\title{
PERK Signaling Promotes Mitochondrial Elongation By Remodeling Membrane Phosphatidic Acid
}

Valerie Perea ${ }^{1}$, Christian Cole ${ }^{2,5}$, Justine Lebeau, ${ }^{1,5}$, Vivian Dolina ${ }^{1}$, Kelsey R. Baron ${ }^{1}$, Aparajita Madhavan ${ }^{1}$, Jeffery W. Kelly ${ }^{2,3}$, Danielle A. Grotjahn ${ }^{4}$, and R. Luke Wiseman ${ }^{1, *}$

${ }^{1}$ Department of Molecular Medicine, Scripps Research, La Jolla, CA

${ }^{2}$ Department of Chemistry, Scripps Research, La Jolla, CA

${ }^{3}$ Skaggs Institute for Chemical Biology, Scripps Research, La Jolla, CA

${ }^{4}$ Department of Integrative, Structural, and Computational Biology, Scripps Research, La Jolla, CA ${ }^{5}$ These authors contributed equally.

${ }^{*}$ To whom correspondences should be addressed:

R. Luke Wiseman

Department of Molecular Medicine

Scripps Research

La Jolla, CA 92037

Email: wiseman@scripps.edu

Phone: (858) 784-8820

Keywords: unfolded protein response (UPR); mitochondrial morphology; phosphatidic acid; endoplasmic reticulum stress 


\section{SUMMARY}

Endoplasmic reticulum (ER) stress and mitochondrial dysfunction are linked in the onset and pathogenesis of numerous diseases. This has led to considerable interest in defining the mechanisms responsible for regulating mitochondria during ER stress. The PERK signaling arm of the unfolded protein response (UPR) has emerged as a prominent ER stress-responsive signaling pathway that regulates diverse aspects of mitochondrial biology. Here, we show that PERK activity also promotes adaptive remodeling of mitochondrial membrane phosphatidic acid (PA) to induce protective mitochondrial elongation during ER stress. We find that PERK activity is required for ER stress-dependent increases in both cellular PA and YME1L-dependent degradation of the intramitochondrial PA transporter PRELID1. Together, these processes lead to the accumulation of PA on the outer mitochondrial membrane where it induces mitochondrial elongation by inhibiting mitochondrial fission. Our results establish a new role for PERK in the adaptive remodeling of mitochondrial phospholipids and demonstrate that PERK-dependent PA regulation functions to adapt organellar shape in response to ER stress. 


\section{INTRODUCTION}

Endoplasmic reticulum (ER) and mitochondrial function are coordinated through the inter-organellar transport of metabolites such as lipids and $\mathrm{Ca}^{2+} \cdot{ }^{1-3}$ As a consequence of this coordination, ER stress can be transmitted to mitochondria and promote mitochondrial dysfunctions implicated in the pathophysiology of numerous diseases including diabetes, cardiovascular disorders, and many neurodegenerative diseases. ${ }^{4-16}$ This pathologic relationship between ER stress and mitochondria has led to significant interest in identifying the stress-responsive signaling pathways responsible for regulating mitochondria in response to ER insults.

The PERK arm of the unfolded protein response (UPR) has emerged as a prominent stress-responsive signaling pathway involved in regulating mitochondria during ER stress. ${ }^{17-19}$ PERK is an ER transmembrane protein that is activated in response to ER stress through a mechanism involving oligomerization and autophosphorylation of its cytosolic kinase domain (Fig. 1A). ${ }^{20-22}$ Activated PERK selectively phosphorylates serine 51 of the $\alpha$ subunit of eukaryotic initiation factor 2 (elF2 $\alpha$ ). Phosphorylated elF2 $\alpha$ prevents formation of ribosomal initiation complexes leading to global mRNA translational attenuation, which functions to reduce the load of newly synthesized proteins during ER stress. ${ }^{20-22}$ PERK-dependent elF2a phosphorylation also leads to the selective translation and activation of transcription factors, such as ATF4, through upstream open reading frames (uORFs) in the promoters of these genes. ${ }^{20-23}$ ATF4 regulates the expression of several stressresponsive genes including redox factors, amino acid biosynthesis genes, the elF2 $\alpha$ phosphatase PPP1R15A/GADD34, and the pro-apoptotic transcription factor DDIT3/CHOP. ${ }^{23-25}$ Through this combination of translational attenuation and transcriptional signaling, PERK promotes both adaptive and pro-apoptotic signaling in response to varying levels and extents of ER stress. ${ }^{20-31}$

PERK localizes to ER-mitochondrial contact sites, positioning this protein to coordinate regulation of these two organelles in response to cellular insults. ${ }^{32}$ Consistent with this, PERK signaling regulates diverse aspects of mitochondrial proteostasis and function in response to stress. PERK regulates mitochondrial protein import, biogenesis, and cristae remodeling in brown adipocytes in response to cold exposure or betaadrenergic stimulation. ${ }^{33,34}$ Further, the PERK-regulated transcription factor ATF4 increases mitochondrial respiratory chain activity during ER stress or nutrient deprivation through a mechanism involving SCAF1dependent increases in supercomplex formation. ${ }^{35}$ ATF4 also regulates the expression of numerous mitochondrial proteostasis factors including the mitochondrial HSP70 HSPA9 and the AAA+ quality control 
protease LONP1 to increase mitochondrial proteostasis capacity during ER stress. ${ }^{25,36}$ Further, PERKdependent translational attenuation regulates mitochondrial protein import by selectively decreasing protein concentrations of the core TIM23 subunit Tim17A, a process dependent on the mitochondrial AAA+ protease YME1L. ${ }^{37}$

PERK signaling also promotes mitochondrial elongation downstream of elF2 $\alpha$ phosphorylationdependent translational attenuation. ${ }^{38}$ This increase in mitochondrial length functions to protect mitochondria during ER stress by preventing premature fragmentation and regulating mitochondrial respiratory chain activity. ${ }^{38}$ However, the mechanistic basis of PERK-dependent mitochondrial elongation was previously undefined. Here, we show that PERK induces mitochondrial elongation through the adaptive remodeling of mitochondrial membrane phosphatidic acid (PA). Our results suggest a model whereby PERK signaling both increases total mitochondrial PA and inhibits trafficking of PA to the inner mitochondrial membrane. This leads to the accumulation of PA on the outer mitochondrial membrane where it induces mitochondrial elongation by inhibiting mitochondrial fission. These results define a new role for PERK in regulating the amount and localization of mitochondrial membrane phospholipids and show that this remodeling is important for adapting mitochondrial morphology during ER stress. 


\section{RESULTS}

\section{Hypomorphic PERK variants inhibit ER stress induced mitochondrial elongation}

Pharmacologic inhibition of PERK signaling blocks mitochondrial elongation induced by ER stress. ${ }^{38}$ Here, we further probed the dependence of ER stress induced mitochondrial elongation on PERK activity in Perk ${ }^{-1}$ MEFs. ${ }^{24}$ We transfected Perk ${ }^{+/+}$or Perk ${ }^{-/}$MEFs with mitochondrial targeted GFP ( $\left.{ }^{\mathrm{mt}} \mathrm{GFP}\right)$ and monitored mitochondrial morphology in cells treated with either the ER stressor thapsigargin (Tg; a SERCA inhibitor) or the translation inhibitor cycloheximide $(\mathrm{CHX})$ - the latter of which induces mitochondrial elongation independent of PERK signaling. ${ }^{38,39}$ Perk $^{-/}$MEFs showed increases in fragmented mitochondria in the absence of treatment (Fig. 1B,C). Tg-induced mitochondrial elongation was also impaired in Perk-deficient cells. However, $\mathrm{CHX}$ treatment reduced mitochondrial fragmentation in Perk-deficient cells, indicating that these cells are not deficient in their ability to increase length in response to reduced translation. Reconstitution of $P$ erk ${ }^{-1}$ MEFs with wild-type PERK restored basal mitochondrial morphology and rescued Tg-induced mitochondrial elongation. However, a hypomorphic PERK haplotype implicated in progressive supranuclear palsy (PSP; PERK $\left.{ }^{\text {PSP }}\right)^{40,41}$ did not significantly impact basally fragmented mitochondria or rescue Tg-induced mitochondrial elongation in Perk-deficient cells. We confirmed similar expression of PERK ${ }^{\mathrm{WT}}$ and PERK ${ }^{\mathrm{PSP}}$ in Perk $^{-/-} \mathrm{MEFs}^{\mathrm{by}}$ immunoblotting (Fig. S1). These results further implicate PERK signaling in ER stress induced mitochondrial elongation and demonstrate that genetic disruptions in PERK signaling impair the regulation of mitochondrial morphology in the presence or absence of ER stress.

\section{Overexpression of cytosolic PA lipases inhibits ER stress induced mitochondrial elongation.}

Mitochondrial morphology is defined by the relative activities of GTPases localized to the inner and outer mitochondrial membranes that regulate organellar fission and fusion. These include the pro-fission GTPase DRP1 of the outer mitochondrial membrane (OMM) and the pro-fusion GTPases MFN1 and MFN2 of the OMM and OPA1 of the inner mitochondrial membrane (IMM). ${ }^{42-46}$ Stress-induced changes in mitochondrial shape are primarily dictated through posttranslational regulation of these GTPases to alter the relative activities of fusion and fission pathways. ${ }^{42-46}$ Previous results indicate that PERK signaling does not significantly influence the posttranslational regulation of these GTPases ${ }^{38}$, suggesting that ER stress induced mitochondrial elongation proceeds through an alternative mechanism. 
Mitochondrial elongation can be induced by the accumulation of saturated PA on the OMM through mechanisms including PA-dependent inhibition of the pro-fission GTPase DRP $1 .{ }^{47-51}$ Interestingly, PERK was previously shown to increase cellular PA during ER stress through a mechanism dependent on PERK kinase activity, but not signaling downstream of elF2 $\alpha$ phosphorylation. ${ }^{52}$ We confirmed that treatment with $\mathrm{Tg}$ increases PA in mitochondria-enriched fractions and whole cell extracts from MEF cells measured by mass spectrometry, biochemical assays, and ELISA (Fig. 2A,B, Fig. S2A,D-E). Another phospholipid, phosphatidylcholine (PC) was not affected in enriched mitochondria (Fig. 2A). Similar results were observed in HeLa cells (Fig. S2B,C). Co-treatment with the PERK inhibitor GSK2606414, a compound that directly inhibits PERK kinase activity (Fig. 1A) ${ }^{53}$, reduced Tg-dependent increases of PA in both MEF and HeLa cells (Fig. 2B, Fig. S2A-C). This indicates that ER stress-dependent increases in PA requires PERK kinase activity, as previously reported. ${ }^{52}$ However, co-treatment of MEF cells with Tg and ISRIB, a compound that blocks PERK signaling downstream of elF2 $\alpha$ phosphorylation (Fig. 1A) ${ }^{54}$, did not appear to mitigate ER stress induced PA increases in mitochondria enriched fractions or whole cell extracts (Fig. S2D,E). This is consistent with previous results suggesting that ER stress increases PA through a mechanism selectively dependent on PERK kinase activity, relative to signaling downstream of elF2 $\alpha$ phosphorylation. ${ }^{52}$

We next determined the dependence of PERK-regulated mitochondrial elongation on PA by monitoring mitochondrial morphology in Tg-treated HeLa cells co-overexpressing ${ }^{\mathrm{mt}}$ GFP and Lipin1 - a cytosolic PA lipase that converts PA to diacylglycerol (DAG). ${ }^{48,49,55,56}$ We confirmed that Lipin1 overexpression reduced cellular PA (Fig. S2F). Overexpression of wild-type, but not catalytically inactive, Lipin1 increased basal mitochondrial fragmentation and inhibited Tg-induced mitochondrial elongation (Fig 2C,D). Similar results were observed in cells treated with CHX. Importantly, Lipin1 overexpression did not significantly influence increases of ATF4 protein, the expression of ATF4 target genes (e.g., Asns, Chop), or TIM17A degradation in Tg-treated cells (Fig. S2G,H). This indicates that Lipin1 overexpression did not influence upstream PERK signaling (Fig. 1A), but instead inhibited ER stress induced mitochondrial elongation at a step downstream of PERK-dependent elF2 $\alpha$ phosphorylation. Overexpression of PA-PLA1 - a cytosolic lipase that converts PA to lysophosphatidic acid $^{49}$ - similarly inhibited mitochondrial elongation in cells treated with $\mathrm{Tg}$ or $\mathrm{CHX}$ without impacting other aspects of PERK signaling (Fig. S2I-L). Collectively, these results indicate that genetic depletion of PA blocks ER stress induced mitochondrial elongation, implicating PA in this process. 


\section{ER stress prevents DRP1-dependent mitochondrial fragmentation.}

Accumulation of PA on the OMM promotes mitochondrial elongation by inhibiting the pro-fission GTPase DRP1. ${ }^{51}$ This was previously demonstrated by showing that genetically increasing PA on the OMM by overexpressing mitoPLD - an OMM lipase that converts cardiolipin to PA - basally increased mitochondrial elongation and inhibited DRP1-dependent mitochondrial fragmentation induced by the uncoupler carbonyl cyanide m-chlorophenylhydrazone (CCCP). ${ }^{51,57}$ Consistent with this, we confirmed that mitoPLD overexpression in HeLa cells increased basal mitochondrial elongation and inhibited CCCP-induced mitochondrial fragmentation (Fig. S3A,B). Pretreatment with $\mathrm{Tg}$ similarly reduced CCCP-induced mitochondrial fragmentation. However, Tg-pretreatment also blocked CCCP-induced proteolytic cleavage of the inner membrane GTPase OPA1 (Fig. S3C) - a biological process upstream of DRP1-dependent mitochondrial fragmentation induced by membrane uncoupling. ${ }^{42-46,58}$ This appears to result from Tg-dependent increases in mitochondrial membrane polarity, which prevent efficient mitochondrial uncoupling in CCCP-treated cells (Fig. S3D). Regardless, the Tg-dependent inhibition of OPA1 processing in cells treated with CCCP precluded our ability to determine whether ER stress inhibits DRP1 activity under these conditions.

To circumvent this problem, we monitored mitochondria morphology in MEF ${ }^{\mathrm{mtGFP}}$ cells pretreated with $\mathrm{Tg}$ and then challenged with ionomycin - a $\mathrm{Ca}^{2+}$ ionophore that increases cytosolic $\mathrm{Ca}^{2+} .{ }^{42-46,59}$ Increases in cytosolic $\mathrm{Ca}^{2+}$ induced by short $(<30 \mathrm{~min})$ treatments with ionomycin or Tg promotes DRP1-dependent mitochondrial fragmentation through a mechanism independent of membrane uncoupling or OPA1 processing. ${ }^{59}$ However, pretreatment for $3 \mathrm{~h}$ with $\mathrm{Tg}$ - a timepoint sufficient to increase PA and induce mitochondrial elongation - inhibits ionomycin-induced mitochondrial fragmentation (Fig. 3A,B). This inhibition is reversed by co-treatment with ISRIB, a small molecule that blocks elF2 $\alpha$ phosphorylation-dependent signaling downstream of PERK (Fig. 1A), confirming that this effect can be attributed to PERK signaling and not dysregulation of intracellular $\mathrm{Ca}^{2+}$ induced by the combined treatment of $\mathrm{Tg}$ and ionomycin. These results are consistent with a model whereby ER stress promotes mitochondrial elongation through a mechanism involving inhibition of DRP1 activity, as was reported previously for mitoPLD overexpression ${ }^{51}$. 
Overexpression of mitoPLD increases cellular PA to similar extents to that observed in Tg-treated cells (Fig. S3E). This provides an opportunity to determine whether increases in PA are sufficient to induce the mitochondrial elongation observed during ER stress. To test this, we monitored mitochondrial morphology in HeLa cells overexpressing mitoPLD and treated with or without Tg. Despite similar increases in PA (Fig. S3E), mitoPLD overexpression did not increase mitochondrial elongation to the same extent observed upon $\mathrm{Tg}$ treatment (Fig. 3C,D). Further, treatment with $\mathrm{Tg}$ increased mitochondrial elongation in mitoPLD overexpressing cells, as compared to untreated controls (Fig. 3C,D). This increase was blocked by cotreatment with the PERK signaling inhibitor ISRIB, indicating that this enhanced elongation could be attributed to PERK signaling downstream of elF2 $\alpha$ phosphorylation (Figs. 1A, 3C,D). Overexpression of mitoPLD did not significantly impact other aspects of PERK signaling in Tg-treated cells such as TIM17A degradation (Fig. S3F), suggesting that this effect cannot be attributed to altered or increased PERK activity. These results suggest that PERK-dependent increases in PA, on its own, may not be sufficient to explain the increase in mitochondrial elongation observed during ER stress and that other PERK-regulated activities induced downstream of elF2 $\alpha$ phosphorylation are also likely involved in this process.

\section{PERK signaling leads to reductions in the intramitochondrial PA transporter PRELID1 during ER}

\section{stress.}

ER stress induced mitochondrial elongation is inhibited by shRNA-depletion of YME1L in HeLa cells. ${ }^{38}$ This suggests a potential role for YME1L in this process. We further confirmed the dependence of Tg-induced mitochondrial elongation on YME1L in MEF ${ }^{\text {mtGFP }}$ cells where Yme1/ was deleted by CRISPR (Fig. S4A,B). PRELID1, an intermembrane space protein that transports PA from the OMM to the IMM (Fig. 4A) ${ }^{55,56}$, is a known substrate of YME1L. ${ }^{60-62}$ Interestingly, PRELID1 is a short-lived protein whose levels are highly sensitive to translational attenuation. ${ }^{63}$ Consistent with this, PRELID1 levels are rapidly reduced in MEF ${ }^{\mathrm{mtGFP}}$ cells treated with $\mathrm{CHX}$ (Fig. S4C). This CHX-dependent reduction in PRELID1 was blocked in Yme1/-deficient cells (Fig. 4B), confirming that PRELID1 is degraded by YME1L under these conditions. Identical results were observed for TIM17A, another short-lived mitochondrial protein degraded by YME1L downstream of translation inhibition (Fig. 4B and Fig. S4C). ${ }^{37}$ 
The sensitivity of PRELID1 to translational attenuation suggests that this protein could similarly be reduced by PERK-regulated translational attenuation. As expected, PRELID1 was rapidly decreased in MEF $^{\text {mtGFP }}$ cells treated with the ER stressor Tg (Fig. 4C). Tg-dependent reductions in PRELID1 were inhibited in cells deficient in Yme1l, indicating that YME1L was required for this process (Fig. 4C). Co-treatment with either the PERK kinase inhibitor GSK2606414 or the PERK signaling inhibitor ISRIB (Fig. 1A) blocked Tgdependent reductions in PRELID1 (Fig. 4D). Similar results were observed for TIM17A. Further, Tg-dependent reductions in PRELID1 and TIM7A were also inhibited in Perk ${ }^{-/-}$MEFs (Fig. 4E). Reconstitution of Perk ${ }^{-/}$with $\mathrm{PERK}^{\mathrm{WT}}$, but not hypomorphic PERK ${ }^{\mathrm{PSP}}$, restored Tg-induced degradation of these proteins. Importantly, CHX reduced PRELID1 and TIM17A in all genotypes, confirming that these proteins remain sensitive to translational attenuation even when PERK signaling is impaired (Fig. 4E). Interestingly, Tg-dependent reductions in PRELID1 were not inhibited in cells deficient in Atf4 (Fig. S4D) ${ }^{64}$, a primary upstream transcription factor in the PERK pathway (Fig. 1A), indicating that this phenotype is independent of PERK-regulated transcriptional signaling. Collectively, these results suggest that PRELID1, like TIM17A ${ }^{37}$, is reduced during ER stress through a YME1L-dependent mechanism downstream of PERK-dependent translational attenuation.

\section{Depletion of PRELID1 rescues ER stress-dependent mitochondrial elongation in cells deficient in}

\section{PERK signaling.}

Decreases in PRELID1 will slow trafficking of PA from the OMM to the IMM (Fig. 4A). This would increase PA on the OMM where it could promote mitochondrial elongation through the inhibition of DRP1. To define the importance of PERK-dependent reductions in PRELID1 on ER stress induced mitochondrial elongation, we used shRNA to deplete Prelid1 from MEF ${ }^{\text {mtGFP }}$ cells and monitored mitochondrial morphology following treatment with $\mathrm{Tg}$. We confirmed PRELID1 depletion by immunoblotting and demonstrated that loss of PRELID1 did not influence ER stress-induced, PERK-dependent mechanisms such as TIM17A degradation

(Fig. S5A). Prelid1 depletion did not basally influence mitochondrial morphology or inhibit Tg-induced mitochondrial elongation (Fig. 5A,B). This indicates that reduction of PRELID1, on its own, is not sufficient to increase mitochondrial elongation, likely reflecting the importance of PERK kinase-dependent increases in PA in this process. ${ }^{52}$ Consistent with this, co-treatment with the PERK kinase inhibitor GSK2606414 blocked Tginduced mitochondrial elongation in Prelid1-deficient cells (Fig. 5A,B). However, PRELID1 depletion partially 
bioRxiv preprint doi: https://doi.org/10.1101/2022 02.23.481593; this version posted February 23, 2022. The copyright holder for this preprint

(which was not certified by peer review) is the author/funder, who has granted bioRxiv a license to display the preprint in perpetuity. It is made available under aCC-BY 4.0 International license.

rescued mitochondrial elongation in cells co-treated with $\mathrm{Tg}$ and ISRIB - an inhibitor that blocks PERK signaling downstream of PERK kinase activity (Fig. 1A), but does not significantly inhibit ER stress dependent increases in mitochondrial PA (Fig. S2D,E). Identical results were observed in HeLa cells depleted of PRELID1 and treated with Tg, GSK2606414, or ISRIB (Fig. 5C,D, Fig. S5B). These results implicate YME1L-dependent reductions of PRELID1 in the PERK-regulated mitochondrial elongation induced during ER stress. 


\section{DISCUSSION}

Mitochondrial elongation is an adaptive mechanism that protects mitochondria in response to diverse pathologic insults. ${ }^{38,65-71}$ Interestingly, numerous different mechanisms have been shown to promote mitochondrial elongation in response to different types of stress. For example, the accumulation of lysophosphatidic acid (LPA) on the outer mitochondrial membrane increases mitochondrial elongation through a MTCH2-dependent mechanism during starvation. ${ }^{69}$ Alternatively, HDAC6-dependent deacetylation of profusion GTPase MFN1 increases mitochondrial length during glucose deprivation by enhancing the activity of organellar fusion pathways. ${ }^{70}$ Further, PKA-dependent phosphorylation or PARKIN-dependent ubiquitination of the pro-fission GTPase DRP1 inhibits mitochondrial fission and promotes mitochondrial elongation under a variety of different conditions. ${ }^{71-73}$ Despite these differences in mechanism, mitochondrial elongation similarly functions to prevent premature fragmentation, regulate mitochondria respiratory chain activity, and promote cell survival in response to diverse pathologic insults, including ER stress. ${ }^{38,65-73}$

ER stress promotes mitochondrial elongation through a process regulated by the PERK arm of the UPR. ${ }^{38}$ Here, we demonstrate that this PERK-dependent increase in elongated mitochondria is attributed to PERK-dependent remodeling of mitochondrial membrane PA (Fig. 6). Previously, ER stress was shown to increase cellular PA through a mechanism dependent on PERK kinase activity, but not elF2 $\alpha$ phosphorylation. ${ }^{52}$ This was suggested to involve direct, PERK-dependent phosphorylation of diacylglycerol (DAG; Fig. 6, step 1). ${ }^{52}$ Our results support the dependence of ER stress induced increases of PA on PERK kinase activity, showing that the PERK kinase inhibitor GSK2606414 reduces Tg-dependent increases of PA more efficiently than ISRIB, a compound that inhibits PERK signaling downstream of PERK kinase activity

(Fig. 1A).

However, our findings also suggest that PERK-dependent increases in PA are insufficient to drive the full extent of mitochondrial elongation observed during ER stress. To account for this, we show that ER stressdependent increases in mitochondrial elongation also involve reductions in the intra-mitochondrial PA trafficking protein PRELID1 (Fig. 6, step 2). Consistent with previous results showing that PERK-dependent mitochondrial elongation requires PERK-dependent translational attenuation ${ }^{38}$, we show that PRELID1 is a short-lived mitochondrial protein that is degraded through a YME1L-dependent mechanism downstream of elF2 $\alpha$ phosphorylation-dependent reductions in translation. Intriguingly, we implicated PERK-dependent 
reductions in PRELID1 in ER stress induced mitochondrial elongation by showing that genetic depletion of PRELID1 partially rescues mitochondrial elongation in cells co-treated with the PERK signaling inhibitor ISRIB, but not the PERK kinase inhibitor GSK2606414. This highlights an important role for reductions in PRELID1, induced downstream of PERK-dependent translational attenuation through a YME1L-dependent mechanism, in the adaptive remodeling of mitochondrial membrane PA observed during ER stress.

The combination of PERK-dependent increases in total PA and YME1L-dependent decreases of PRELID1 should increase PA on the OMM during conditions of ER stress. Previous studies have shown that increases in OMM PA promote mitochondrial elongation through multiple mechanisms including direct inhibition of the pro-fission GTPase DRP1 (Fig. 6, step 3). ${ }^{51}$ Consistent with an important role for OMM PA in ER stress induced mitochondrial elongation, we show that overexpression of two different cytosolic PA lipases, Lipin1 and PA-PLA1, block mitochondrial elongation observed in Tg-treated cells. Further, we demonstrate that pre-treatment with the ER stressor $\mathrm{Tg}$ inhibits DRP1-dependent mitochondrial fragmentation in ionomycintreated cells. Collectively, these results support a model whereby PERK-dependent increases in OMM PA promote mitochondrial elongation through a mechanism involving reductions in mitochondrial fission, likely mediated through the direct inhibition of the pro-fission GTPase DRP1 (Fig. 6).

PERK-regulated translational and transcriptional signaling regulate diverse aspects of mitochondrial proteostasis and function. Our results provide new insights into PERK-dependent remodeling of mitochondria by demonstrating that signaling through this UPR pathway promotes adaptive remodeling of mitochondrial membrane PA to induce protective organelle elongation during ER stress. As we and others continue studying the impact of PERK signaling on mitochondrial biology, additional mitochondrial pathways regulated through PERK signaling will also likely be identified, further expanding our understanding of the critical role for this stress-responsive signaling pathway in adapting mitochondria during ER stress. Moving forward, it will also be interesting to define how different PERK-dependent mitochondrial adaptations integrate to influence other aspects of mitochondrial function during conditions of stress. For example, recent cryo-electron tomography results indicate that mitochondrial elongation correlates with cristae remodeling in ER stressed cells, suggesting that these changes to bulk mitochondrial morphology and ultrastructure may be coordinated. ${ }^{74}$ Further, PRELID1-dependent trafficking of PA to the IMM is a critical step in the biosynthesis of cardiolipins - a mitochondrial-specific phospholipid important for regulating membrane morphology and multiple mitochondrial 
functions including respiratory chain activity and apoptotic signaling. ${ }^{48}$ While acute ER insults are unlikely to significantly influence cardiolipin levels owing to the higher stability of this phospholipid as compared to other membrane phospholipids ${ }^{75}$, chronic PERK-dependent translational attenuation, and subsequent sustained reductions in PRELID1, could lead to reductions in cardiolipin that impact mitochondrial energy metabolism and cell fate. Thus, an improved understanding of how different PERK-dependent alterations to mitochondrial morphology and function integrate will provide additional insight to the critical importance of this pathway in regulating mitochondria during conditions of ER stress.

The global importance of PERK in adapting mitochondria during ER stress also suggests that disruptions in this signaling could exacerbate mitochondrial dysfunction in disease. Genetic mutations in EIF2AK3, the gene that encodes PERK, are causatively associated with Wolcott-Rallison syndrome - a devastating disease characterized by early onset diabetes, skeletal deformities, and growth impairments. ${ }^{76}$ Further, a hypomorphic PERK haplotype is associated with tauopathies including progressive supranuclear palsy (PSP) and late stage Alzheimer's disease. ${ }^{15,40,41}$ Interestingly, mitochondrial dysfunction has been implicated in all these disorders, suggesting that failure of PERK-dependent mitochondrial regulation is a contributing factor in disease pathogenesis. Consistent with this, we show that hypomorphic PSP-associated PERK alleles disrupt PERK-dependent mitochondrial elongation and YME1L-dependent PRELID1 degradation. In contrast, chronic PERK activation is also implicated in the pathogenesis of numerous neurodegenerative diseases involving mitochondrial dysfunction such as AD and prion disease. ${ }^{77-81}$ While the specific importance of PERK signaling on mitochondrial function in these diseases remains largely undefined, this suggests that PERK signaling, while adaptive during acute ER insults, could become detrimental to mitochondria in response to chronic ER insults potentially through mechanisms such as reductions in cardiolipin. Further investigations will be required to determine the specific impact of altered PERK signaling on mitochondria morphology and function in the context of these diseases to reveal both the pathologic and potentially therapeutic implications of PERK activity on the mitochondrial dysfunction observed in the pathogenesis of these disorders. 
bioRxiv preprint doi: https://doi.org/10.1101/2022.02.23.481593; this version posted February 23, 2022. The copyright holder for this preprint (which was not certified by peer review) is the author/funder, who has granted bioRxiv a license to display the preprint in perpetuity. It is made available under aCC-BY 4.0 International license. 


\section{MATERIALS AND METHODS}

Cell Culture, Transfections, Lentiviral Transduction, and CRISPR deletion

MEF $^{\text {mtGFP }}$ (a kind gift from Peter Schultz, The Scripps Research Institute; TSRI) ${ }^{82}$, Perk ${ }^{+/+}$and Perk $^{-/-}$MEFs $^{24}$, Atf4 $^{+/+}$and Atf4 $^{-/-}$MEFs ${ }^{64}$, HeLa cells (purchased from the ATCC), or HEK293T cells were cultured in DMEM (Corning-Cellgro) supplemented with 10\% fetal bovine serum (FBS; Omega Scientific), 2 mM L-glutamine (GIBCO), $100 \mathrm{U} / \mathrm{mL}$ penicillin, and $100 \mathrm{mg} / \mathrm{mL}$ streptomycin (GIBCO). Cells were maintained at $37^{\circ} \mathrm{C}$ and $5 \%$ $\mathrm{CO}_{2}$. Non-essential amino acids (GIBCO) and 2-mercaptoethanol were also added to culture media of Atf4 $^{+/+}$ and Atf4 $^{-/}$MEFs and Perk ${ }^{+/+}$and Perk ${ }^{+/+}$MEFs. HeLa cells were transfected by calcium phosphate precipitation, as previously described..$^{38}$ MEF cells were transfected with MEF Avalanche Transfection Reagent (EZ Biosystems) according to the manufacturer's protocol. Lentivirus were prepared by transfecting HEK293T cells with pRSV-rev (Addgene \#12253), pMDL-RRE (Addgene, \#12251), pMD2.6 (Addgene \#12259), and with the indicated shRNA in the pLKO.1 vector (Sigma) using calcium phosphate precipitation. After $24 \mathrm{~h}$, the transfection media was removed and replaced with complete DMEM and incubated overnight for viral production. Virus containing media was removed the following day and filtered with a $0.45 \mu \mathrm{m}$ syringe filter (Genessee Scientific). Polybrene (Sciquest/Fisher) was added to the viral containing media at a concentration of $10 \mu \mathrm{g} / \mathrm{mL}$ and the media was then added to HeLa or MEF ${ }^{\mathrm{mtGFP}}$ cells. Stable pools of cells expressing nonsilencing or gene-specific knockdowns were then generated through selection with puromycin $(3 \mathrm{mg} / \mathrm{mL}$ for MEF cells and $1 \mathrm{mg} / \mathrm{mL}$ for HeLa). Knockdown was then confirmed by immunoblotting. Yme1/ was deleted from MEF ${ }^{\text {mtGFP }}$ cells using CRISPR/Cas9. Briefly, cells were transfected with plasmid pSpCas9(BB)-2A-Puro (PX459; $\quad$ Addgene, \#62988) containing sgRNA $\quad$ against $\quad$ Yme1/ (GATCCAATATGAGATGTATGCCAAC AAACGTTGGCATACATCTCATATT) using MEF Avalanche, following manufacturers protocols. After transfection, cells were selected with puromycin and single clones were screened for YME1L depletion by qPCR and immunoblotting.

Plasmids, shRNAs, and compounds.

HA-LIPIN1 ${ }^{\text {WT }}$, HA-LIPIN ${ }^{\text {Mut }}$, and mitoPLD-GFP overexpression constructs were kind gifts from Hiromi Sesaki (Johns Hopkins) and described previously. ${ }^{51}$ The PA-PLA1-GFP overexpression construct was from Addgene (\#162880). Perk ${ }^{W T}$ and Perk ${ }^{P S P}$ overexpression plasmids were kind gifts from Jonathan Lin (Stanford). 
Plasmids containing shRNA were purchased from Sigma in the pLKO.1 vector: mouse Prelid1 shRNA (TRCN0000345802), human PRELID1 shRNA (TRCN0000130829). All compounds used in this study were purchased: thapsigargin (Tg; Fisher Scientific), GSK2606414 (BioVision Inc.), ISRIB (Sigma), CCCP (Sigma), and ionomycin (Sigma).

\section{Fluorescence Microscopy.}

HeLa cells transfected with ${ }^{\mathrm{mt}}$ GFP or MEF ${ }^{\mathrm{mtGFP}}$ cells were seeded at a density of 100,000 cells/well on glassbottom dishes (MatTek) coated with poly-D-lysine (Sigma) or rat tail collagen 1 (GIBCO). Cells were then treated as indicated and images were recorded with an Olympus IX71 microscope with 60x oil objective (Olympus), a Hamamatsu C8484 camera (Hamamatsu Photonics), and HCl image software (Hamamatsu Photonics). At least 20 cells were imaged per condition for each experiment for quantification. Quantification was performed by blinding the images and then scoring cells based on the presence of primarily fragmented, tubular, or elongated mitochondria, as before ${ }^{38}$. Three different researchers scored each set of images and these scores were averaged for each individual experiment. All quantifications shown were performed for at least 3 independent experiments, where averages in morphology quantified from each individual experiment were then combined. The data were then prepared in PRISM (GraphPad, San Diego, CA) and plotted on a stacked bar plot to show the average morphology and standard error of the mean across all experiments. Statistical comparisons were performed using a 2-way ANOVA in PRISM, comparing the relative amounts of fragmented, tubular, or elongated mitochondria across different conditions.

\section{Phospholipid Quantification}

For MS analysis of PA, whole cell pellets were resuspended in $500 \mu$ l of a cold hypotonic buffer consisting of 1 mM PBS, pH 7.4. The material was then homogenized on ice using a glass Dounce homogenizer (30 strokes). The homogenized sample was centrifuged at $500 \mathrm{~g}$ for $4 \mathrm{~min}$ then the supernatant was transferred to a $1.5-\mathrm{ml}$ microfuge tube and lyophilized overnight. The lyophilized material was weighed and normalized by total mass prior to performing a modified Bligh and Dyer extraction. ${ }^{83}$ The proceeding steps were done with glass pipettes and tubes to avoid plastic contamination. PA was extracted by the addition of $100 \mu \mathrm{l} / \mathrm{mg}$ of cold methanol containing an internal PA standard (Splash Lipidomix, Avanti) at a dilution of (1:50), followed by $50 \mu \mathrm{l} / \mathrm{mg}$ of 
cold chloroform $\left(\mathrm{CHCl}_{3}\right)$ with occasional vortex mixing. Milli-Q $\mathrm{H}_{2} \mathrm{O}$ containing $5 \mathrm{mM}$ erythorbate was added at a volume of $50 \mu \mathrm{l} / \mathrm{mg}$. The sample was agitated and centrifuged in glass test tubes at $200 \mathrm{~g}$ for $10 \mathrm{~min}$. The bottom phase was collected in a clean test tube, while the upper phase was re-extracted two additional times with methanol: $\mathrm{CHCl}_{3}(1: 1, \mathrm{v}: \mathrm{v})$ containing $\mathrm{HCL}$ at final concentration of $10 \mathrm{mM}$. The organic phases were combined and dried under vacuum to afford a lipid film that was stored at $-80^{\circ} \mathrm{C}$ until $\mathrm{MS}$ analysis. Mitochondria enriched fractions were processed similar to whole cell samples except they were normalized via protein concentration as determined by the Pierce ${ }^{\mathrm{TM}}$ BCA Protein Assay kit (Thermo Scientific). In Brief, PA was extracted from the mitochondria enriched fractions using $10 \mu \mathrm{l} / \mu \mathrm{g}$ methanol, $5 \mu \mathrm{l} / \mu \mathrm{g} \mathrm{CHCl}{ }_{3}$ and $5 \mu \mathrm{l} / \mu \mathrm{g}$ milli-Q $\mathrm{H}_{2} \mathrm{O}$ containing $5 \mathrm{mM}$ erythorbate as previously described above.

Extracted lipid samples and a PA external standard (SPLASH Lipidomix, Avanti) were removed from the $-80^{\circ} \mathrm{C}$ freezer and resuspended in $100 \mu \mathrm{l}$ of methanol. Negative mode LC-MS analysis was performed on an Agilent 6230 ESI-TOF-MS System calibrated with a reference solution at $\mathrm{m} / \mathrm{z}$ 1033.9881. A XBridge BEH C8 XP Column (Waters, $2.5 \mu \mathrm{m}, 4.6 \mathrm{~mm} \times 150 \mathrm{~mm}$ ) was used at a flow rate of $500 \mu \mathrm{L} / \mathrm{min}$, employing the following gradient: 30 to $100 \%$ solvent B over $30 \mathrm{~min}, 100 \%$ isocratic B for $10 \mathrm{~min}$ followed by a return to $30 \%$ B for 5 min. Solvents A consisted of MilliQ water and methanol (9:1, v:v). Solvents B consisted of acetonitrile: 2-propanol (5:3, v:v), and both contained $10 \mathrm{mM}$ Piperidine, $10 \mathrm{mM}$ ammonium acetate and $0.1 \%$ formic acid. Prior to processing, raw.d files were converted to the open format mzXML using MSConvert software, which is part of the ProteoWizard software toolkit. ${ }^{84}$ Mass detection was achieved using mzmine 2 wavelet algorithm, ADAP chromatogram builder and ADAP deconvolution ${ }^{85}$, which are part of the MZmine 2 software package. ${ }^{86}$ Initial lipid identifications were achieved using lipidmaps database with a $\mathrm{m} / \mathrm{z}$ tolerance of $10 \mathrm{ppm}$, subsequently PA detected lipids were filtered out for further processing. These putative peaks were validated by aligning RT to the internal and external standards followed by graphical identification of PA lipids by plotting the Kendrick mass defect plot employing $\mathrm{CH}_{2}$ as the repeating unit. The quantification of all PA lipid class was normalized based on the abundance of the internal standard PA (15:0-18:1-d7-PA), which factors in extraction efficiency and sample handling. Total PA levels were then normalized to vehicle for the indicated number of independent experiments.

For quantification of PA by ELISA, MEF or HeLa cells were treated as indicated and collected on ice and then lysed with $20 \mathrm{mM}$ Hepes pH 7.4, $100 \mathrm{mM} \mathrm{NaCal}, 1 \mathrm{mM}$ EDTA, 1\% Triton X100) supplemented with 
Pierce protease inhibitor (ThermoFisher). Protein concentrations for each sample were then quantified using the Bio-Rad Bradford assay. PA was then measured using the Human Phosphatidic Acid Antibody (IgM ELISA Kit (Lifeome Bioloabs) following the manufacturers protocol and monitoring fluorescence on a Tecan F250Pro microplate reader (Tecan).

For quantification of PA by fluorometric biochemical assay, MEF or HeLa cells were treated as indicated and collected on ice and then centrifuged and washed with cold PBS three times. Samples were then sonicated using the Misonix S-4000 sonicator then processed and PA was measured according the manufacturer's protocol for the Total Phosphatidic Assay Kit (CellBio Labs).

\section{Immunoblotting and Antibodies}

Whole cells were lysed at room temperature in HEPES lysis buffer (20 mM Hepes pH 7.4, $100 \mathrm{mM} \mathrm{NaCal,} 1$ mM EDTA, 1\% Triton X100) supplemented with 1x Pierce protease inhibitor (ThermoFisher). Total protein concentrations of lysates were then normalized using the Bio-Rad protein assay and lystaes were combined with 1x Laemmli buffer supplemented with $100 \mathrm{mM}$ DTT and boiled for $5 \mathrm{~min}$. Samples were then separated by SDS-PAGE and transferred to nitrocellulose membranes (Bio-Rad). Membranes were then blocked with 5\% milk in tris-buffered saline (TB) and then incubated overnight at $4^{\circ} \mathrm{C}$ with the indicated primary antibody. The next day, membranes were washed in TBS supplemented with Tween, incubated with the species appropriate secondary antibody conjugated to IR-Dye (LICOR Biosciences) and then imaged using an Odyssey Infrared Imaging System (LICOR Biosciences). Quantification was then carried out using the LICOR Imaging Studio software.

Primary antibodies were acquired from commercial sources and used in the indicated dilutions in antibody buffer (50mM Tris $[\mathrm{pH} 7.5], 150 \mathrm{mM} \mathrm{NaCl}$ supplemented with $5 \% \mathrm{BSA}(\mathrm{w} / \mathrm{v})$ and $\left.0.1 \% \mathrm{NaN}_{3}(\mathrm{w} / \mathrm{v})\right)$ : Tim17a (Thermo Scientific, 1:1000), PRELID1 [aa27-54] (Sciquest, 1:1000), YME1L (Abgent, 1:1000), ATF4 (Cell Signaling, 1:500), Tubulin [B-5-1-2] (Sigma, 1:5000), Tim23 (BD Transduction Labs, 1:1000), HSP60 [LK1] (Thermo Scientific, 1:1000), PERK (Sciquest, 1:1000), HA [Clone: 16B12] (Biolegend, 1: 1000), GFP (B2) (Sciquest, 1:1000), OPA1 (BD Transduction Labs, 1:2000). 
The relative mRNA expression of target genes was measured using quantitative RT-PCR. Cells were treated as indicated and then washed with phosphate buffered saline (PBD; Gibco). RNA was extracted using QuickRNA MiniPrepKit (Zymo Research) according to the manufacturers protocol. RNA (500 ng) was then converted to cDNA using the QuantiTect Reverse Transcription Kit (Qiagen). qPCR reactions were prepared using Power SYBR Green PCR Master Mix (Applied Biosystems), and primers (below) were obtained from Integrated DNA Technologies. Amplification reactions were run in an ABI 7900HT Fast Real Time PCR machine with an initial melting period of $95^{\circ} \mathrm{C}$ for $5 \mathrm{~min}$ and then 45 cycles of $10 \mathrm{~s}$ at $95^{\circ} \mathrm{C}, 30 \mathrm{~s}$ at $60^{\circ} \mathrm{C}$.

Primers used in this study:

Human ASNS: forward: GCAGCTGAAAGAAGCCCAAG; reverse: AGCCTGAATGCCTTCCTCAG

Human CHOP/DDI3: forward: ACCAAGGGAGAACCAGGAAACG; reverse TCACCATTCGGTCAATCAGAGC

Human HSPA5/BIP: forward: GCCTGTATTTCTAGACCTGCC; reverse, TTCATCTTGCCAGCCAGTTG

Human RIBOP: forward: CGT CGC CTC CTA CCT GCT; reverse, CCA TTC AGC TCA CTG ATA ACC TTG

\section{Membrane Depolarization}

MEFs were seeded at a density of 85,000 cells/well of a 6 -well plate and treated with $500 \mathrm{nM}$ Tg for $3 \mathrm{~h}$ prior to collection. CCCP $(10 \mu \mathrm{M})$ was added 50 min before collection, followed by $200 \mathrm{nM}$ TMRE (Thermofisher) 20 min before collection. Samples were collected using TrypLE Express and cell culture media. Following a brief centrifugation, cell pellets were washed in DPBS (Gibco) and resuspended in DPBS supplemented with 5\% BSA. Fluorescence intensity of TMRE for 20,000 cells/condition was recorded on the PE channel of a BD Biosciences LSR II analytical flow cytometer. Data are presented as geometric mean of the fluorescence intensity from three experiments normalized to vehicle-treated cells.

\section{Statistical Analysis}

Statistics were calculated in PRISM 9 (GraphPad, San Diego, CA). Data are presented as mean +/- SEM and were analyzed by 2-way ANOVA with Tukey's multiple correction test or the appropriate student t-tests, as indicated in the accompanying figure legends. Indications of non-significant interactions from 2-way ANOVA were generally omitted for clarity. 


\section{ACKNOWLEDGEMENTS.}

We thank Jessica Rosarda and Cristina Puchades for experiments and analysis related to this work. We thank Benjamin Barad, Michaela Medina, Enrique Saez, and Katja Lamia for critical reading of this manuscript. This work was funded by the National Institutes of Health (NS095892 to RLW), a National Science Foundation predoctoral fellowship (VP), and the George E. Hewitt Postdoctoral fellowship (VD).

\section{CONFLICT OF INTEREST STATEMENT.}

We declare no conflicts related to the work described in this manuscript.

\section{AUTHOR CONTRIBUTIONS.}

VP, CC, and JL conceived, designed, performed, and interpreted the experiments. VD, KB, and AM performed experiments. DG and RLW interpreted experiments. RLW supervised the project. VP and RLW wrote the manuscript. VP, CC, JL, VD, KB, JWK, DG, and RLW provided critical revisions to the manuscript and approval for submission. 


\section{REFERENCES}

1 Csordas, G., Weaver, D. \& Hajnoczky, G. Endoplasmic Reticulum-Mitochondrial Contactology: Structure and Signaling Functions. Trends Cell Biol 28, 523-540, doi:10.1016/j.tcb.2018.02.009 (2018).

2 Wu, H., Carvalho, P. \& Voeltz, G. K. Here, there, and everywhere: The importance of ER membrane contact sites. Science 361, doi:10.1126/science.aan5835 (2018).

3 Rowland, A. A. \& Voeltz, G. K. Endoplasmic reticulum-mitochondria contacts: function of the junction. Nat Rev Mol Cell Biol 13, 607-625, doi:10.1038/nrm3440 (2012).

4 Rocha, M., Apostolova, N., Diaz-Rua, R., Muntane, J. \& Victor, V. M. Mitochondria and T2D: Role of Autophagy, ER Stress, and Inflammasome. Trends Endocrinol Metab 31, 725-741, doi:10.1016/j.tem.2020.03.004 (2020).

Ren, J., Bi, Y., Sowers, J. R., Hetz, C. \& Zhang, Y. Endoplasmic reticulum stress and unfolded protein response in cardiovascular diseases. Nat Rev Cardiol 18, 499-521, doi:10.1038/s41569-021-00511-w (2021).

Area-Gomez, E. et al. Upregulated function of mitochondria-associated ER membranes in Alzheimer disease. EMBO J 31, 4106-4123, doi:10.1038/emboj.2012.202 (2012).

Brown, M. K. \& Naidoo, N. The endoplasmic reticulum stress response in aging and age-related diseases. Front Physio/ 3, 263, doi:10.3389/fphys.2012.00263 (2012).

De Strooper, B. \& Scorrano, L. Close encounter: mitochondria, endoplasmic reticulum and Alzheimer's disease. EMBO J 31, 4095-4097, doi:10.1038/emboj.2012.279 (2012).

9 Hughes, D. \& Mallucci, G. R. The unfolded protein response in neurodegenerative disorders therapeutic modulation of the PERK pathway. FEBS J 286, 342-355, doi:10.1111/febs.14422 (2019). Liu, M. \& Dudley, S. C., Jr. Role for the Unfolded Protein Response in Heart Disease and Cardiac Arrhythmias. Int J Mol Sci 17, doi:10.3390/ijms17010052 (2015).

11 Morris, G. et al. The Endoplasmic Reticulum Stress Response in Neuroprogressive Diseases: Emerging Pathophysiological Role and Translational Implications. Mol Neurobiol 55, 8765-8787, doi:10.1007/s12035-018-1028-6 (2018).

12 Rodriguez-Arribas, M. et al. Mitochondria-Associated Membranes (MAMs): Overview and Its Role in Parkinson's Disease. Mol Neurobiol 54, 6287-6303, doi:10.1007/s12035-016-0140-8 (2017).

13 Schon, E. A. \& Area-Gomez, E. Mitochondria-associated ER membranes in Alzheimer disease. Mol Cell Neurosci 55, 26-36, doi:10.1016/j.mcn.2012.07.011 (2013).

14 Smith, H. L. \& Mallucci, G. R. The unfolded protein response: mechanisms and therapy of neurodegeneration. Brain 139, 2113-2121, doi:10.1093/brain/aww101 (2016).

15 Stutzbach, L. D. et al. The unfolded protein response is activated in disease-affected brain regions in progressive supranuclear palsy and Alzheimer's disease. Acta Neuropathol Commun 1, 31, doi:10.1186/2051-5960-1-31 (2013).

16 Xiang, C., Wang, Y., Zhang, H. \& Han, F. The role of endoplasmic reticulum stress in neurodegenerative disease. Apoptosis 22, 1-26, doi:10.1007/s10495-016-1296-4 (2017).

17 Rainbolt, T. K., Saunders, J. M. \& Wiseman, R. L. Stress-responsive regulation of mitochondria through the ER unfolded protein response. Trends Endocrinol Metab 25, 528-537, doi:10.1016/j.tem.2014.06.007 (2014).

18 Quintana-Cabrera, R. \& Soriano, M. E. ER Stress Priming of Mitochondrial Respiratory suPERKomplex Assembly. Trends Endocrinol Metab 30,685-687, doi:10.1016/j.tem.2019.08.003 (2019).

19 Cannon, B. \& Nedergaard, J. A PERKy way to make mitochondrial cristae. Trends Endocrinol Metab 32, 417-419, doi:10.1016/j.tem.2021.04.003 (2021).

20 Walter, P. \& Ron, D. The unfolded protein response: from stress pathway to homeostatic regulation. Science 334, 1081-1086, doi:10.1126/science.1209038 (2011).

21 Hetz, C. \& Papa, F. R. The Unfolded Protein Response and Cell Fate Control. Mol Cell 69, 169-181, doi:10.1016/j.molcel.2017.06.017 (2018).

22 Gardner, B. M., Pincus, D., Gotthardt, K., Gallagher, C. M. \& Walter, P. Endoplasmic reticulum stress sensing in the unfolded protein response. Cold Spring Harb Perspect Biol 5, a013169, doi:10.1101/cshperspect.a013169 (2013).

23 Wek, R. C. \& Cavener, D. R. Translational control and the unfolded protein response. Antioxid Redox Signal 9, 2357-2371, doi:10.1089/ars.2007.1764 (2007). 
24 Harding, H. P., Zhang, Y., Bertolotti, A., Zeng, H. \& Ron, D. Perk is essential for translational regulation and cell survival during the unfolded protein response. Mol Cell 5, 897-904, doi:10.1016/s10972765(00)80330-5 (2000).

25 Han, J. et al. ER-stress-induced transcriptional regulation increases protein synthesis leading to cell death. Nat Cell Biol 15, 481-490, doi:10.1038/ncb2738 (2013).

26 Hetz, C., Zhang, K. \& Kaufman, R. J. Mechanisms, regulation and functions of the unfolded protein response. Nat Rev Mol Cell Biol 21, 421-438, doi:10.1038/s41580-020-0250-z (2020).

27 Halliday, M., Hughes, D. \& Mallucci, G. R. Fine-tuning PERK signaling for neuroprotection. J Neurochem 142, 812-826, doi:10.1111/jnc.14112 (2017).

28 Haucke, V. V. PERKing up the ER stress response. Trends Cell Biol 9, 129, doi:10.1016/s09628924(99)01529-9 (1999).

29 lurlaro, R. \& Munoz-Pinedo, C. Cell death induced by endoplasmic reticulum stress. FEBS J 283, 26402652, doi:10.1111/febs.13598 (2016).

30 Lin, J. H., Li, H., Zhang, Y., Ron, D. \& Walter, P. Divergent effects of PERK and IRE1 signaling on cell viability. PLoS One 4, e4170, doi:10.1371/journal.pone.0004170 (2009).

31 Sovolyova, N., Healy, S., Samali, A. \& Logue, S. E. Stressed to death - mechanisms of ER stressinduced cell death. Biol Chem 395, 1-13, doi:10.1515/hsz-2013-0174 (2014).

Verfaillie, T. et al. PERK is required at the ER-mitochondrial contact sites to convey apoptosis after ROS-based ER stress. Cell Death Differ 19, 1880-1891, doi:10.1038/cdd.2012.74 (2012). Kato, H. et al. ER-resident sensor PERK is essential for mitochondrial thermogenesis in brown adipose tissue. Life Sci Alliance 3, doi:10.26508/lsa.201900576 (2020).

Latorre-Muro, P. et al. A cold-stress-inducible PERK/OGT axis controls TOM70-assisted mitochondria protein import and cristae formation. Cell Metab 33, 598-614 e597, doi:10.1016/j.cmet.2021.01.013 (2021).

Balsa, E. et al. ER and Nutrient Stress Promote Assembly of Respiratory Chain Supercomplexes through the PERK-elF2alpha Axis. Mol Cell 74, 877-890 e876, doi:10.1016/j.molcel.2019.03.031 (2019).

36 Hori, O. et al. Transmission of cell stress from endoplasmic reticulum to mitochondria: enhanced expression of Lon protease. J Cell Biol 157, 1151-1160, doi:10.1083/jcb.200108103 (2002).

37 Rainbolt, T. K., Atanassova, N., Genereux, J. C. \& Wiseman, R. L. Stress-regulated translational attenuation adapts mitochondrial protein import through Tim17A degradation. Cell Metab 18, 908-919, doi:10.1016/j.cmet.2013.11.006 (2013).

38 Lebeau, J. et al. The PERK Arm of the Unfolded Protein Response Regulates Mitochondrial Morphology during Acute Endoplasmic Reticulum Stress. Cell Rep 22, 2827-2836, doi:10.1016/j.celrep.2018.02.055 (2018).

39 Tondera, D. et al. SLP-2 is required for stress-induced mitochondrial hyperfusion. EMBO J 28, 15891600, doi:10.1038/emboj.2009.89 (2009).

40 Hoglinger, G. U. et al. Identification of common variants influencing risk of the tauopathy progressive supranuclear palsy. Nat Genet 43, 699-705, doi:10.1038/ng.859 (2011).

41 Yuan, S. H. et al. Tauopathy-associated PERK alleles are functional hypomorphs that increase neuronal vulnerability to ER stress. Hum Mol Genet 27, 3951-3963, doi:10.1093/hmg/ddy297 (2018).

42 Mishra, P. \& Chan, D. C. Metabolic regulation of mitochondrial dynamics. J Cell Biol 212, 379-387, doi:10.1083/jcb.201511036 (2016).

43 Chan, D. C. Mitochondrial Dynamics and Its Involvement in Disease. Annu Rev Pathol 15, 235-259, doi:10.1146/annurev-pathmechdis-012419-032711 (2020).

44 Fenton, A. R., Jongens, T. A. \& Holzbaur, E. L. F. Mitochondrial dynamics: Shaping and remodeling an organelle network. Curr Opin Cell Biol 68, 28-36, doi:10.1016/j.ceb.2020.08.014 (2020).

45 Sabouny, R. \& Shutt, T. E. Reciprocal Regulation of Mitochondrial Fission and Fusion. Trends Biochem Sci 45, 564-577, doi:10.1016/j.tibs.2020.03.009 (2020).

46 Giacomello, M., Pyakurel, A., Glytsou, C. \& Scorrano, L. The cell biology of mitochondrial membrane dynamics. Nat Rev Mol Cell Biol 21, 204-224, doi:10.1038/s41580-020-0210-7 (2020).

47 Acoba, M. G., Senoo, N. \& Claypool, S. M. Phospholipid ebb and flow makes mitochondria go. J Cell Biol 219, doi:10.1083/jcb.202003131 (2020).

48 Kameoka, S., Adachi, Y., Okamoto, K., lijima, M. \& Sesaki, H. Phosphatidic Acid and Cardiolipin Coordinate Mitochondrial Dynamics. Trends Cell Biol 28, 67-76, doi:10.1016/j.tcb.2017.08.011 (2018). 
49 Baba, T. et al. Phosphatidic acid (PA)-preferring phospholipase A1 regulates mitochondrial dynamics. $J$ Biol Chem 289, 11497-11511, doi:10.1074/jbc.M113.531921 (2014).

$50 \mathrm{Ha}$, E. E. \& Frohman, M. A. Regulation of mitochondrial morphology by lipids. Biofactors 40, 419-424, doi:10.1002/biof.1169 (2014).

51 Adachi, Y. et al. Coincident Phosphatidic Acid Interaction Restrains Drp1 in Mitochondrial Division. Mol Cell 63, 1034-1043, doi:10.1016/j.molcel.2016.08.013 (2016).

52 Bobrovnikova-Marjon, E. et al. PERK utilizes intrinsic lipid kinase activity to generate phosphatidic acid, mediate Akt activation, and promote adipocyte differentiation. Mol Cell Biol 32, 2268-2278, doi:10.1128/MCB.00063-12 (2012).

53 Axten, J. M. et al. Discovery of GSK2656157: An Optimized PERK Inhibitor Selected for Preclinical Development. ACS Med Chem Lett 4, 964-968, doi:10.1021/ml400228e (2013).

54 Sidrauski, C. et al. Pharmacological brake-release of mRNA translation enhances cognitive memory. Elife 2, e00498, doi:10.7554/eLife.00498 (2013).

55 Tatsuta, T. \& Langer, T. Intramitochondrial phospholipid trafficking. Biochim Biophys Acta Mol Cell Biol Lipids 1862, 81-89, doi:10.1016/j.bbalip.2016.08.006 (2017).

56 Tamura, Y., Kawano, S. \& Endo, T. Lipid homeostasis in mitochondria. Biol Chem 401, 821-833, doi:10.1515/hsz-2020-0121 (2020).

$57 \mathrm{Li}, \mathrm{S}$. et al. Transient assembly of F-actin on the outer mitochondrial membrane contributes to mitochondrial fission. J Cell Biol 208, 109-123, doi:10.1083/jcb.201404050 (2015). Jones, E. et al. A threshold of transmembrane potential is required for mitochondrial dynamic balance mediated by DRP1 and OMA1. Cell Mol Life Sci 74, 1347-1363, doi:10.1007/s00018-016-2421-9 (2017).

59 Ji, W. K., Hatch, A. L., Merrill, R. A., Strack, S. \& Higgs, H. N. Actin filaments target the oligomeric maturation of the dynamin GTPase Drp1 to mitochondrial fission sites. Elife 4, e11553, doi:10.7554/eLife.11553 (2015).

60 Potting, C., Wilmes, C., Engmann, T., Osman, C. \& Langer, T. Regulation of mitochondrial phospholipids by Ups1/PRELI-like proteins depends on proteolysis and Mdm35. EMBO J 29, 28882898, doi:10.1038/emboj.2010.169 (2010).

61 Tamura, Y. et al. Role for two conserved intermembrane space proteins, Ups1p and Ups2p, [corrected] in intra-mitochondrial phospholipid trafficking. $J$ Biol Chem 287, 15205-15218, doi:10.1074/jbc.M111.338665 (2012).

62 MacVicar, T. et al. Lipid signalling drives proteolytic rewiring of mitochondria by YME1L. Nature 575, 361-365, doi:10.1038/s41586-019-1738-6 (2019).

$63 \mathrm{Li}, \mathrm{J}$. et al. Proteome-wide mapping of short-lived proteins in human cells. Mol Cell 81, $4722-4735$ e4725, doi:10.1016/j.molcel.2021.09.015 (2021).

64 Harding, H. P. et al. An integrated stress response regulates amino acid metabolism and resistance to oxidative stress. Mol Cell 11, 619-633, doi:10.1016/s1097-2765(03)00105-9 (2003).

65 Gomes, L. C., Di Benedetto, G. \& Scorrano, L. During autophagy mitochondria elongate, are spared from degradation and sustain cell viability. Nat Cell Biol 13, 589-598, doi:10.1038/ncb2220 (2011).

66 Gomes, L. C., Di Benedetto, G. \& Scorrano, L. Essential amino acids and glutamine regulate induction of mitochondrial elongation during autophagy. Cell Cycle 10, 2635-2639, doi:10.4161/cc.10.16.17002 (2011).

67 Lee, J. S. et al. Histone deacetylase inhibitors induce mitochondrial elongation. J Cell Physiol 227, 2856-2869, doi:10.1002/jcp.23027 (2012).

68 Rambold, A. S., Kostelecky, B., Elia, N. \& Lippincott-Schwartz, J. Tubular network formation protects mitochondria from autophagosomal degradation during nutrient starvation. Proc Natl Acad Sci U S A 108, 10190-10195, doi:10.1073/pnas.1107402108 (2011).

69 Labbe, K. et al. The modified mitochondrial outer membrane carrier MTCH2 links mitochondrial fusion to lipogenesis. J Cell Biol 220, doi:10.1083/jcb.202103122 (2021).

70 Lee, J. Y. et al. MFN1 deacetylation activates adaptive mitochondrial fusion and protects metabolically challenged mitochondria. J Cell Sci 127, 4954-4963, doi:10.1242/jcs.157321 (2014).

71 Oshima, Y. et al. Parkin-independent mitophagy via Drp1-mediated outer membrane severing and inner membrane ubiquitination. J Cell Biol 220, doi:10.1083/jcb.202006043 (2021).

72 Cribbs, J. T. \& Strack, S. Reversible phosphorylation of Drp1 by cyclic AMP-dependent protein kinase and calcineurin regulates mitochondrial fission and cell death. EMBO Rep 8, 939-944, doi:10.1038/sj.embor.7401062 (2007). 
73 Chang, C. R. \& Blackstone, C. Cyclic AMP-dependent protein kinase phosphorylation of Drp1 regulates its GTPase activity and mitochondrial morphology. $J$ Biol Chem 282, 21583-21587, doi:10.1074/jbc.C700083200 (2007).

74 Barad, B. A., Medina, M., Fuentes, D., Wiseman, R. L. \& Grotjahn, D. A. A surface morphometrics toolkit to quantify organellar membrane ultrastructure using cryo-electron tomography. bioRxiv (2022).

75 Falabella, M., Vernon, H. J., Hanna, M. G., Claypool, S. M. \& Pitceathly, R. D. S. Cardiolipin, Mitochondria, and Neurological Disease. Trends Endocrinol Metab 32, 224-237, doi:10.1016/j.tem.2021.01.006 (2021).

76 Delepine, M. et al. EIF2AK3, encoding translation initiation factor 2-alpha kinase 3, is mutated in patients with Wolcott-Rallison syndrome. Nat Genet 25, 406-409, doi:10.1038/78085 (2000).

77 Halliday, M. et al. Partial restoration of protein synthesis rates by the small molecule ISRIB prevents neurodegeneration without pancreatic toxicity. Cell Death Dis 6, e1672, doi:10.1038/cddis.2015.49 (2015).

78 Halliday, M. et al. Repurposed drugs targeting elF2\&alpha;-P-mediated translational repression prevent neurodegeneration in mice. Brain 140, 1768-1783, doi:10.1093/brain/awx074 (2017).

79 Moreno, J. A. et al. Sustained translational repression by elF2alpha-P mediates prion neurodegeneration. Nature 485, 507-511, doi:10.1038/nature11058 (2012).

80 Radford, H., Moreno, J. A., Verity, N., Halliday, M. \& Mallucci, G. R. PERK inhibition prevents taumediated neurodegeneration in a mouse model of frontotemporal dementia. Acta Neuropathol 130, 633-642, doi:10.1007/s00401-015-1487-z (2015).

81 Bell, M. C., Meier, S. E., Ingram, A. L. \& Abisambra, J. F. PERK-opathies: An Endoplasmic Reticulum Stress Mechanism Underlying Neurodegeneration. Curr Alzheimer Res 13, 150-163, doi:10.2174/1567205013666151218145431 (2016).

82 Wang, D. et al. A small molecule promotes mitochondrial fusion in mammalian cells. Angew Chem Int Ed Eng/51, 9302-9305, doi:10.1002/anie.201204589 (2012).

83 Bligh, E. G. \& Dyer, W. J. A rapid method of total lipid extraction and purification. Can J Biochem Physiol 37, 911-917, doi:10.1139/059-099 (1959).

84 Chambers, M. C. et al. A cross-platform toolkit for mass spectrometry and proteomics. Nat Biotechnol 30, 918-920, doi:10.1038/nbt.2377 (2012).

85 Myers, O. D., Sumner, S. J., Li, S., Barnes, S. \& Du, X. One Step Forward for Reducing False Positive and False Negative Compound Identifications from Mass Spectrometry Metabolomics Data: New Algorithms for Constructing Extracted Ion Chromatograms and Detecting Chromatographic Peaks. Anal Chem 89, 8696-8703, doi:10.1021/acs.analchem.7b00947 (2017).

86 Pluskal, T., Castillo, S., Villar-Briones, A. \& Oresic, M. MZmine 2: modular framework for processing, visualizing, and analyzing mass spectrometry-based molecular profile data. BMC Bioinformatics 11, 395, doi:10.1186/1471-2105-11-395 (2010). 

available under aCC-BY 4.0 International license.
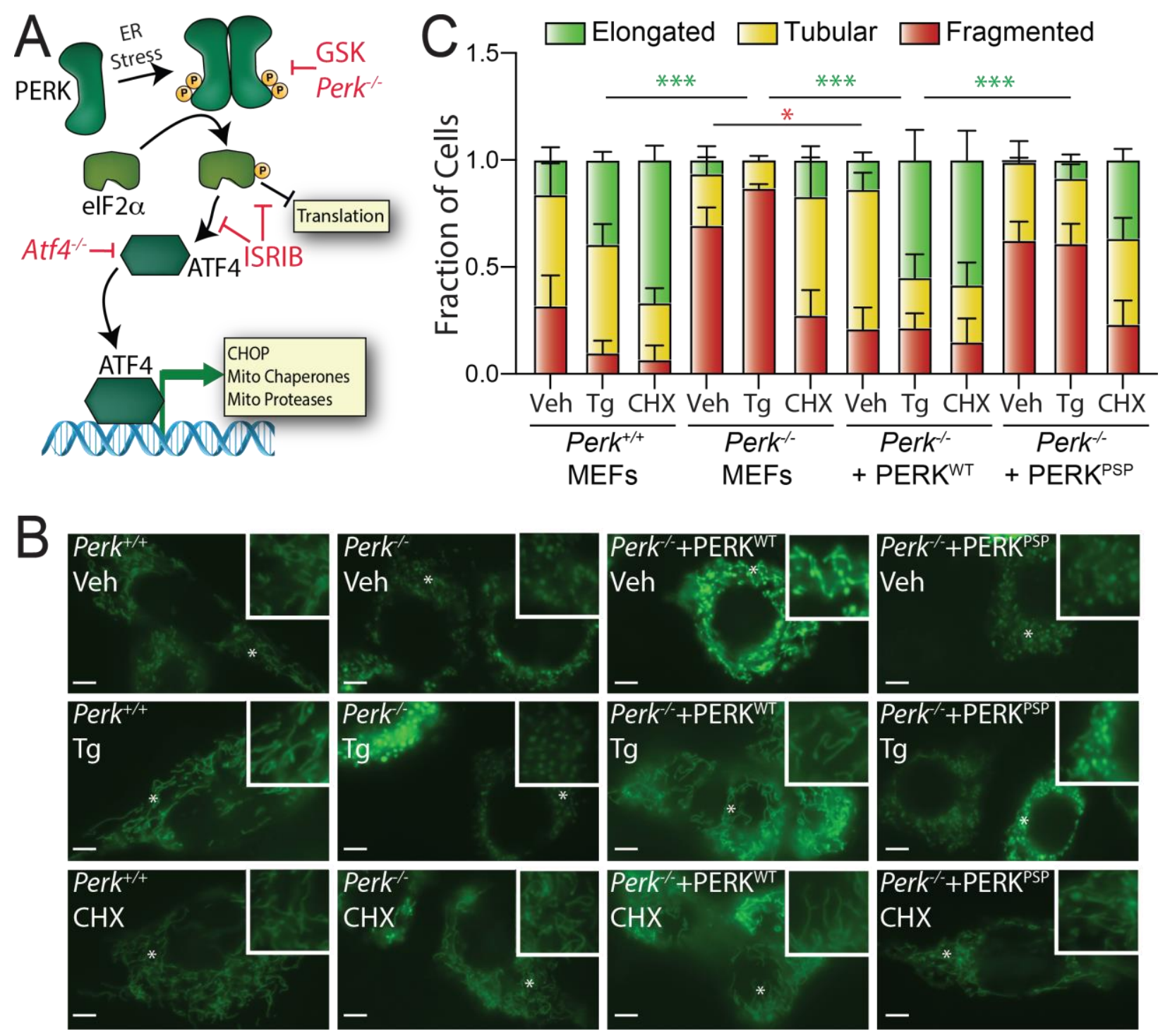

Figure 1. ER stress induced mitochondrial elongation is impaired in cells expressing hypomorphic PERK variants. A. Illustration showing the mechanism of PERK-regulated transcriptional and translational signaling. Specific genetic and pharmacologic manipulations used to disrupt PERK signaling are shown. $\mathbf{B}$. Representative images of Perk ${ }^{+/+}$MEFs, Perk ${ }^{-1}$ MEFs, or Perk $k^{-/}$MEFs transfected with wild-type PERK ${ }^{\mathrm{WT}}$ or the PSP-associated PERK allele (PERK ${ }^{\text {PSP }}$ ) treated for $6 \mathrm{~h}$ with thapsigargin $(\mathrm{Tg} ; 500 \mathrm{nM})$ or cycloheximide (CHX; $50 \mu \mathrm{g} / \mathrm{mL})$. The inset shows 2-fold magnification of the image centered on the asterisk. Scale bars, 5 $\mu \mathrm{m}$. C. Quantification of fragmented (red), tubular (yellow), or elongated (green) mitochondria from the images shown in (B). Error bars show SEM for $n=3$ independent experiments. ${ }^{*} p<0.05,{ }^{* *} \mathrm{p}<0.005$ for 2 -way ANOVA (red indicates comparison between fragmented mitochondria fractions; green indicates comparisons between elongated mitochondria fractions). 

available under aCC-BY 4.0 International license.
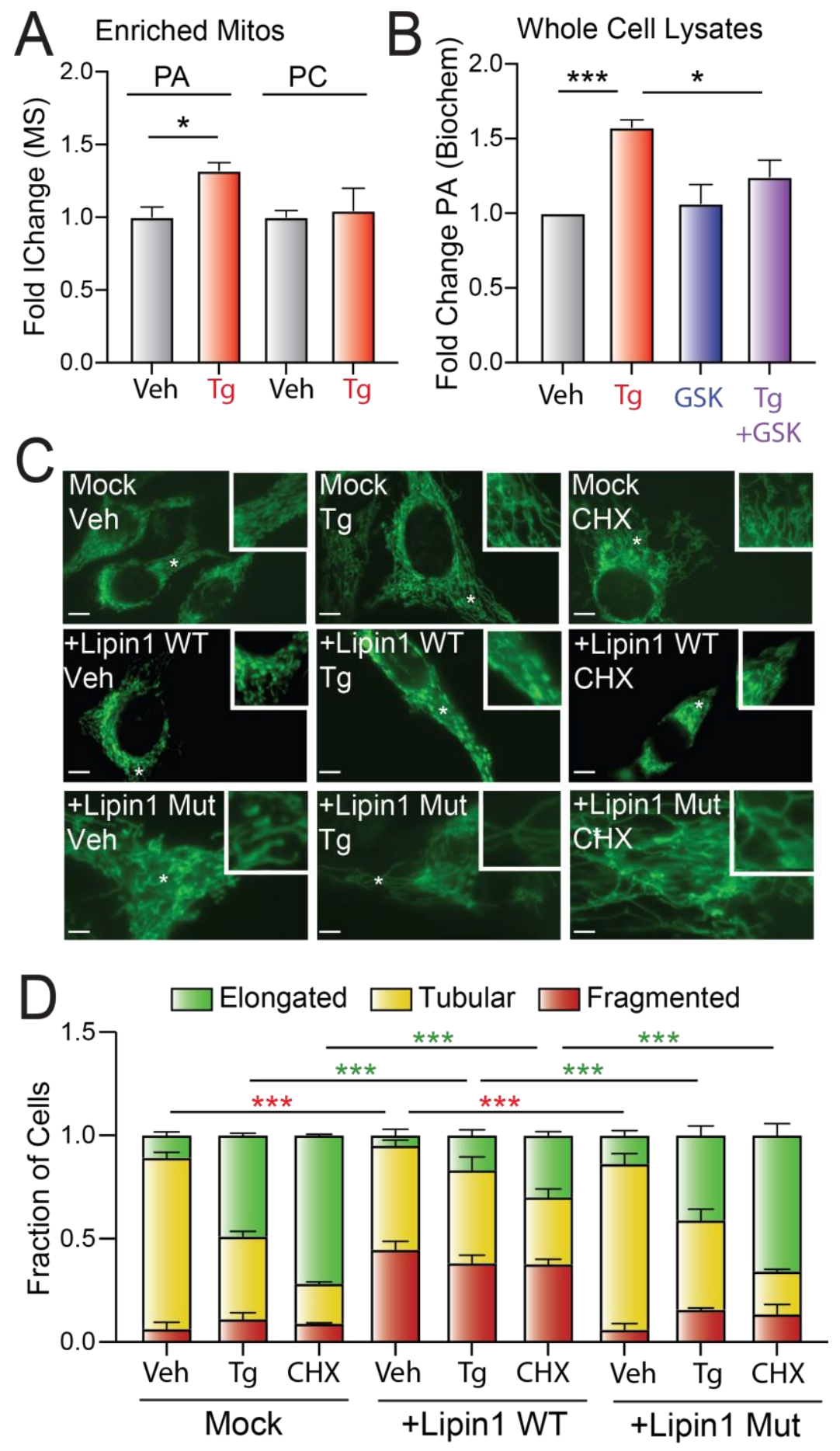

Figure 2. Overexpression of PA lipases inhibits ER stress induced mitochondrial elongation. A. Relative amounts of phosphatidic acid (PA) and phosphatidylcholine (PC), measured by mass spectrometry, in mitochondrial fractions isolated from MEF cells treated for $6 \mathrm{~h}$ with vehicle or thapsigargin (Tg; $500 \mathrm{nM})$. Error bars show SEM for $n=3$ replicates. ${ }^{*} p<0.05$ for unpaired t-test. B. Relative amounts of phosphatidic acid (PA), measured by biochemical assay (see Materials and Methods), in whole cell lysates prepared from MEF cells treated for $3 \mathrm{~h}$ with vehicle or thapsigargin $(\mathrm{Tg} ; 500 \mathrm{nM})$. Error bars show SEM for $\mathrm{n}=3$ replicates. ${ }^{*} \mathrm{p}<0.05$, ${ }^{* * *} p<0.005$ for paired t-test. C. Representative images of HeLa cells expressing ${ }^{\mathrm{mt}} \mathrm{GFP}$ transfected with mock, wild-type Lipin1 (Lipin $1^{\mathrm{wt}}$ ), or catalytically inactive Lipin1 (Lipin1 ${ }^{\mathrm{mut}}$ ) treated for $3 \mathrm{~h}$ with thapsigargin (Tg; 500 $\mathrm{nM})$ or cycloheximide $(\mathrm{CHX} ; 50 \mu \mathrm{g} / \mathrm{mL})$. The inset shows 2-fold magnification of the image centered on the asterisk. Scale bars, $5 \mu \mathrm{m}$. D. Quantification of fragmented (red), tubular (yellow), or elongated (green) mitochondria from the images shown in (D). Error bars show SEM for $n=3$ experiments. $p$-value reflects comparisons of elongated (green) or fragmented (red) mitochondria populations for the indicated conditions. ${ }^{* * *} p<0.005$ for 2-way ANOVA (red indicates comparison between fragmented mitochondria fractions; green indicates comparisons between elongated mitochondria fractions). 


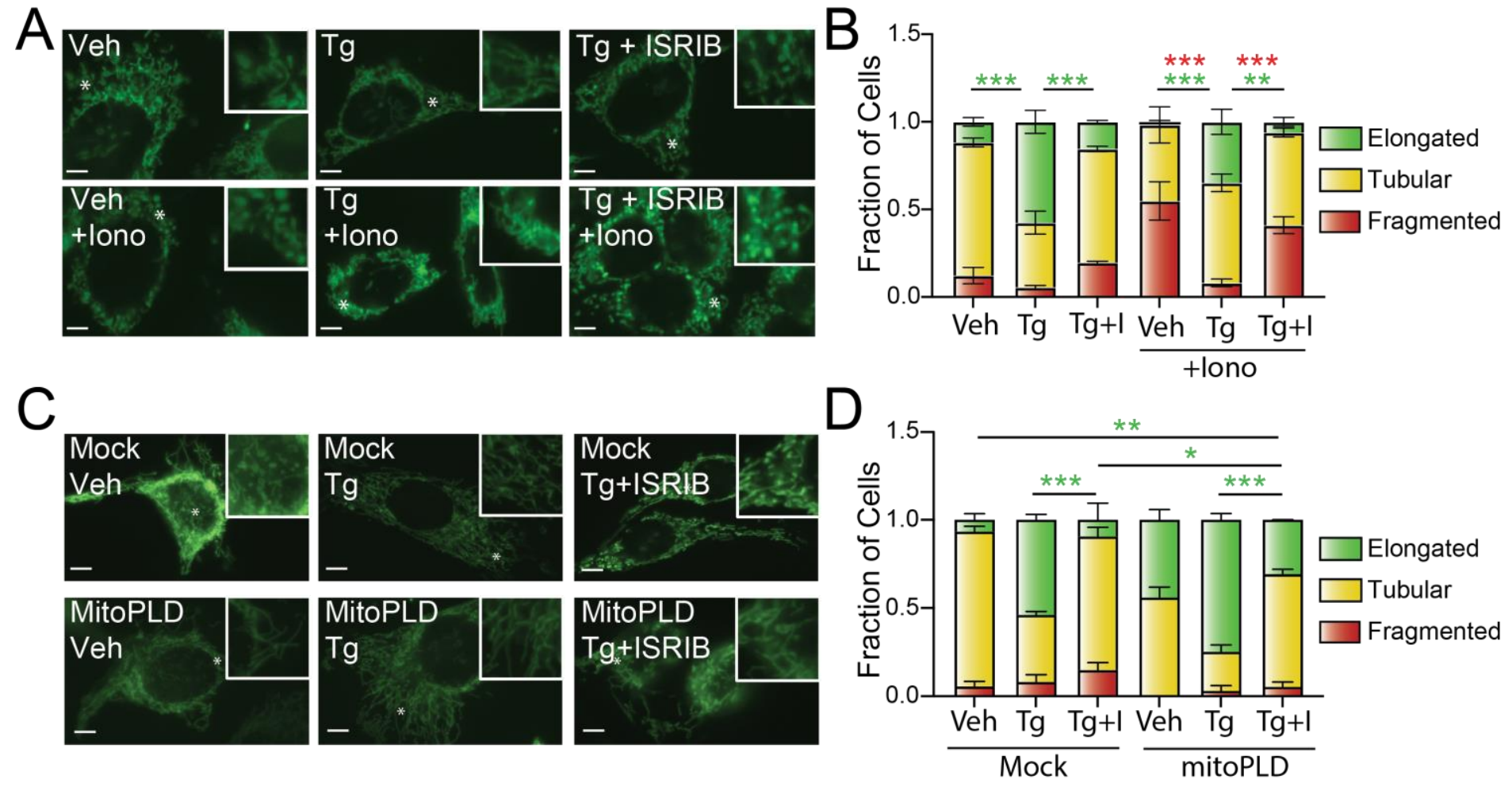

Figure 3. ER stress induced mitochondrial elongation inhibits lonomycin-induced mitochondrial fragmentation. A. Representative images of MEF ${ }^{\mathrm{mtGFP}}$ cells pre-treated for $3 \mathrm{~h}$ with vehicle, thapsigargin (Tg; $500 \mathrm{nM})$, or $\mathrm{Tg}$ and ISRIB $(0.2 \mu \mathrm{M})$ then challenged with vehicle or ionomycin (lono; $1 \mu \mathrm{M})$ for 30 min. The inset shows 2 -fold magnification of the image centered on the asterisk. Scale bars, $5 \mu \mathrm{m}$. B. Quantification of fragmented (red), tubular (yellow), or elongated (green) mitochondria from the images shown in (A). Error bars show SEM for $n=3$ experiments. ${ }^{* *} p<0.01,{ }^{* * *} p<0.005$ for 2 -way ANOVA (red indicates comparison between fragmented mitochondria fractions; green indicates comparisons between elongated mitochondria fractions). C. Representative images of HeLa cells expressing ${ }^{\mathrm{mt}} \mathrm{GFP}$ transfected with mock or mitoPLD ${ }^{\mathrm{GFP}}$ and then treated with vehicle, $\mathrm{Tg}(500 \mathrm{nM})$, or $\mathrm{Tg}+$ ISRIB $(0.2 \mu \mathrm{M})$. The inset shows 2 -fold magnification of the image centered on the asterisk. Scale bars, $5 \mu \mathrm{m}$. Note the expression of mitoPLD ${ }^{\text {GFP }}$ did not impair our ability to accurately monitor mitochondrial morphology in these cells. D. Quantification of fragmented (red), tubular (yellow), or elongated (green) mitochondria from the images shown in (C). Error bars show SEM for $n=3$ experiments. $p$-value reflects comparisons of elongated (green) mitochondria populations for the indicated conditions. ${ }^{*} p<0.05,{ }^{* *} p<0.01,{ }^{* * *} p<0.005$ for 2 -way ANOVA (green indicates comparisons between elongated mitochondria fractions). 


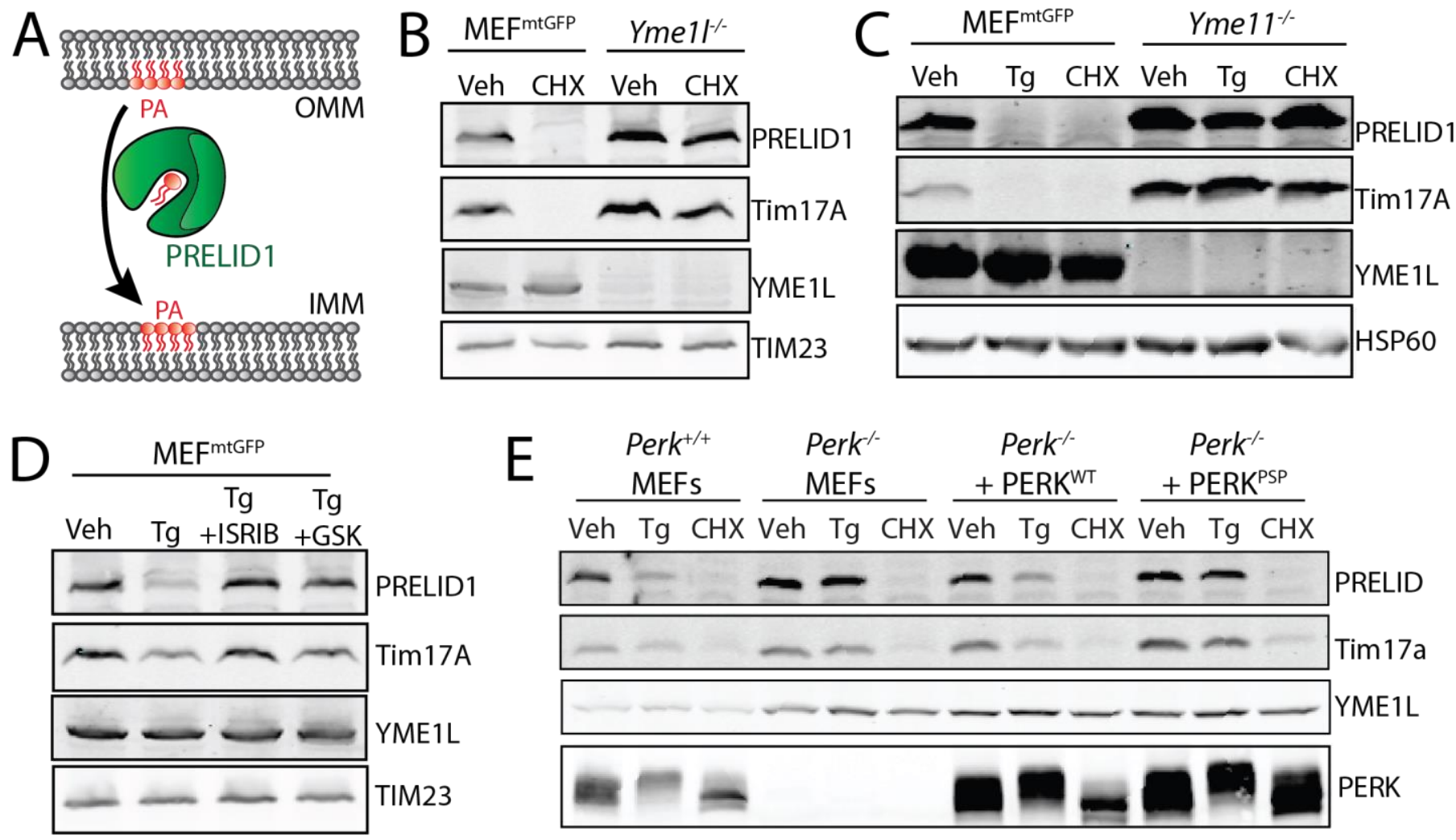

Figure 4. ER stress reduces PRELID1 through a YME1L-dependent mechanism downstream of PERKdependent translational attenuation. A. Illustration showing the PRELID1-dependent trafficking of PA from the outer to inner mitochondrial membranes (OMM and IMM, respectively). B. Immunoblot of lysates prepared from MEF $F^{\text {mtGFP }}$ cells and $Y$ me1/-deficient MEF ${ }^{\text {mtGFP }}$ cells treated for $3 \mathrm{~h}$ with vehicle or cycloheximide (CHX; 50 $\mu \mathrm{g} / \mathrm{mL}$ ). C. Immunoblot of lysates prepared from MEF ${ }^{\text {mGFP }}$ cells and Yme1-deficient MEF ${ }^{\text {mtGFP }}$ cells treated for $3 \mathrm{~h}$ with vehicle, thapsigargin (Tg; $500 \mathrm{nM})$ or cycloheximide (CHX; $50 \mu \mathrm{g} / \mathrm{mL})$. D. Immunoblot of MEF $\mathrm{m}_{\mathrm{m} G \mathrm{FP}}$ cells treated with vehicle, Tg $(500 \mathrm{nM})$, Tg and ISRIB $(0.2 \mu \mathrm{M})$, or Tg and GSK2606414 (GSK; $1 \mu \mathrm{M})$. E. Immunoblot of lysates prepared from Perk ${ }^{+/+}$MEFs, Perk ${ }^{-/}$MEFs, or Perk ${ }^{-/}$MEFs transfected with wild-type PERK $^{\mathrm{WT}}$ or the PSP-associated PERK allele (PERK ${ }^{\text {PSP }}$ ) treated for $6 \mathrm{~h}$ with thapsigargin $(\mathrm{Tg} ; 500 \mathrm{nM}$ ) or cycloheximide (CHX; $50 \mu \mathrm{g} / \mathrm{mL})$. 
bioRxiv preprint doi: https://doi.org/10.1101/2022.02.23.481593; this version posted February 23, 2022. The copyright holder for this preprint (which was not certified by peer review) is the author/funder, who has granted bioRxiv a license to display the preprint in perpetuity. It is made available under aCC-BY 4.0 International license.
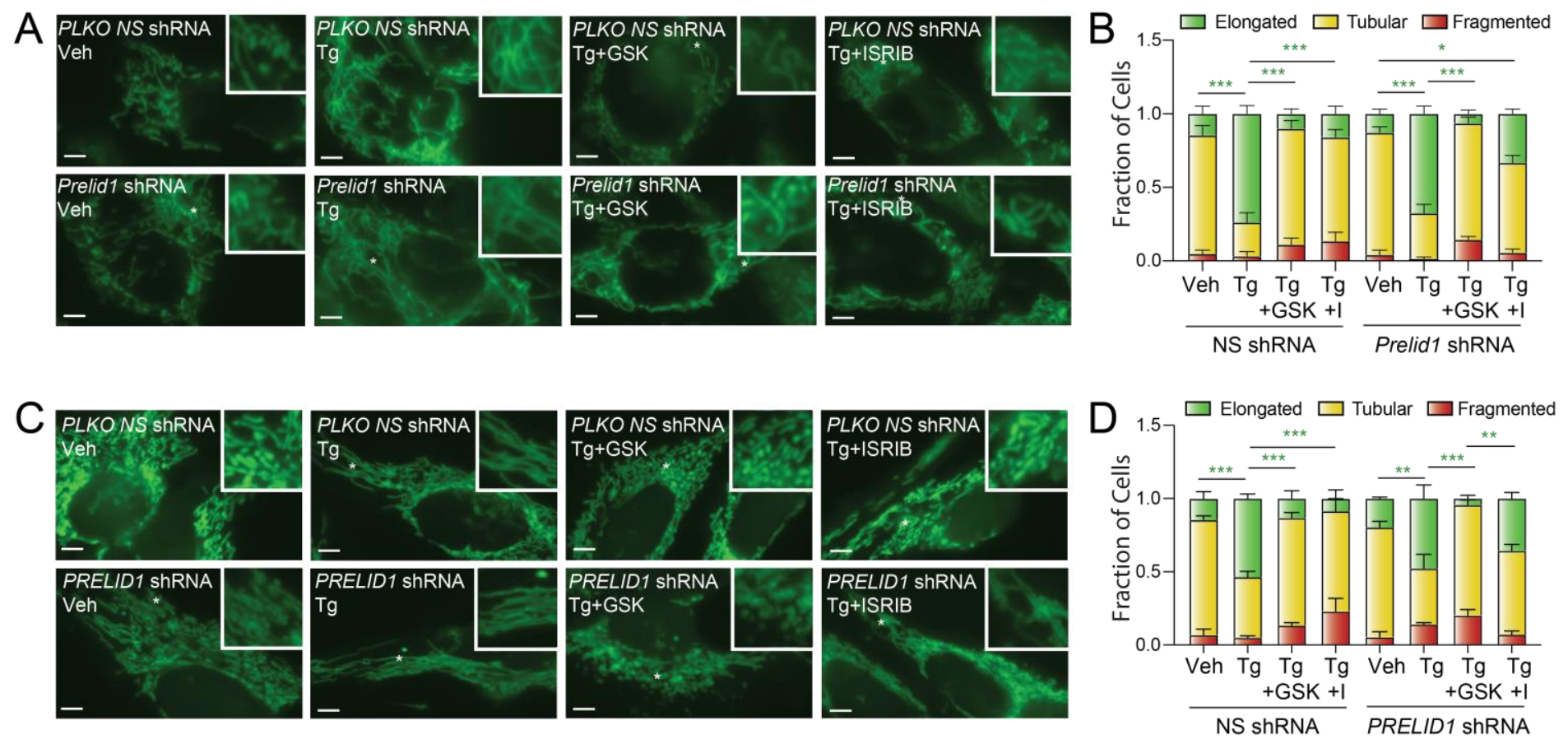

Figure 5. Reductions in PRELID1 contribute to ER stress induced mitochondrial elongation. A. Representative images of MEF ${ }^{\text {mtGFP }}$ cells expressing non-silencing (NS) or Prelid1 shRNA treated for $3 \mathrm{~h}$ with thapsigargin (Tg; $500 \mathrm{nM})$, GSK2606414 (GSK; $1 \mu \mathrm{M})$, and ISRIB $(0.2 \mu \mathrm{M})$, as indicated. The inset shows 2fold magnification of the image centered on the asterisk. Scale bars, $5 \mu \mathrm{m}$. B. Quantification of fragmented (red), tubular (yellow), or elongated (green) mitochondria from the images shown in (A). Error bars show SEM for $n=5$ experiments. ${ }^{*} p<0.05 ;{ }^{* *} p<0.005$ for 2 -way ANOVA (green indicates comparisons between elongated mitochondria fractions). C. Representative images of HeLa cells expressing ${ }^{\mathrm{mt}} \mathrm{GFP}$ and non-silencing (NS) or Prelid1 shRNA treated for $3 \mathrm{~h}$ with thapsigargin (Tg; $500 \mathrm{nM})$, GSK2606414 (GSK; $1 \mu \mathrm{M})$, and ISRIB $(0.2 \mu \mathrm{M})$, as indicated. The inset shows 2 -fold magnification of the image centered on the asterisk. Scale bars, $5 \mu \mathrm{m}$. D. Quantification of fragmented (red), tubular (yellow), or elongated (green) mitochondria from the images shown in (C). Error bars show SEM for $\mathrm{n}=3$ experiments. $\mathrm{p}$-value reflects comparisons of elongated (green) mitochondria populations for the indicated conditions. ${ }^{* *} p<0.01 ;{ }^{* *} p<0.005$ for 2-way ANOVA (green indicates comparisons between elongated mitochondria fractions). 
bioRxiv preprint doi: https://doi org/10.1101/2022 02.23 481593: this version posted February 23,2022 . The copyright holder for this preprint (which was not certified by peer review) is the author/funder, who has granted bioRxiv a license to display the preprint in perpetuity. It is made available under aCC-BY 4.0 International license.

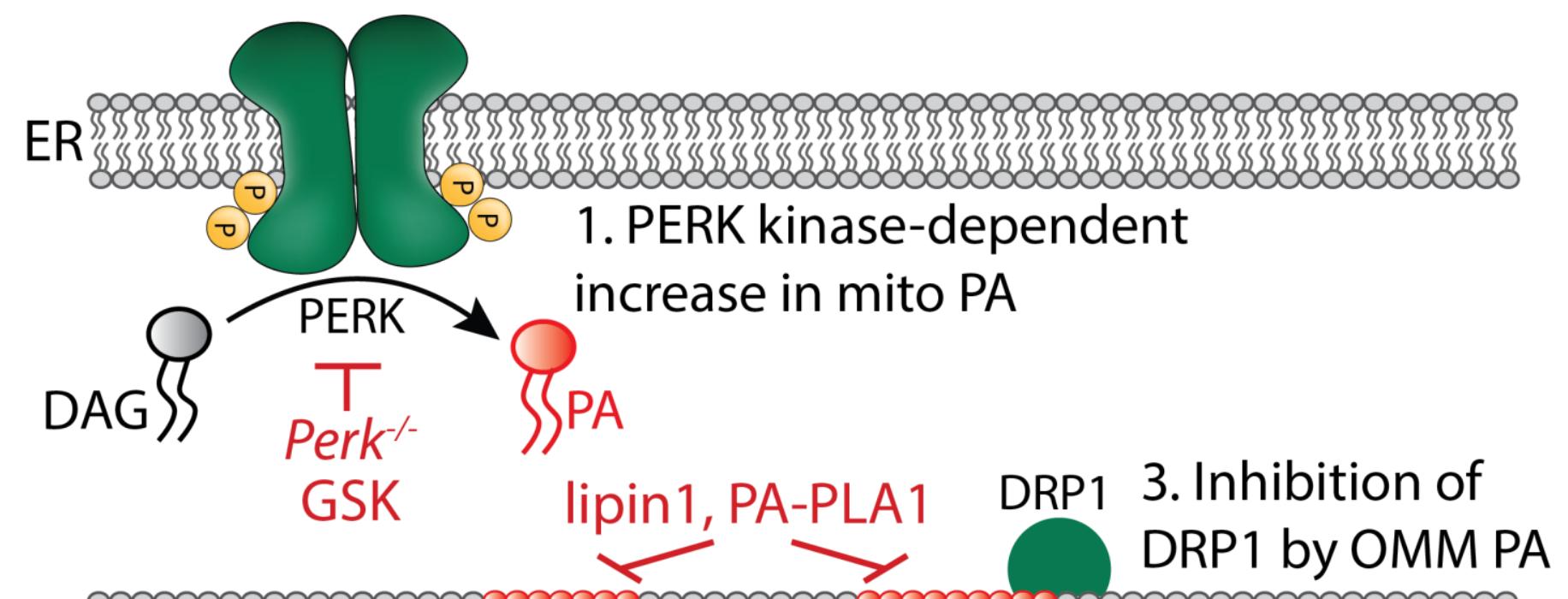

$\mathrm{OMM}$ M

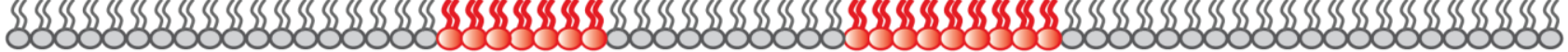
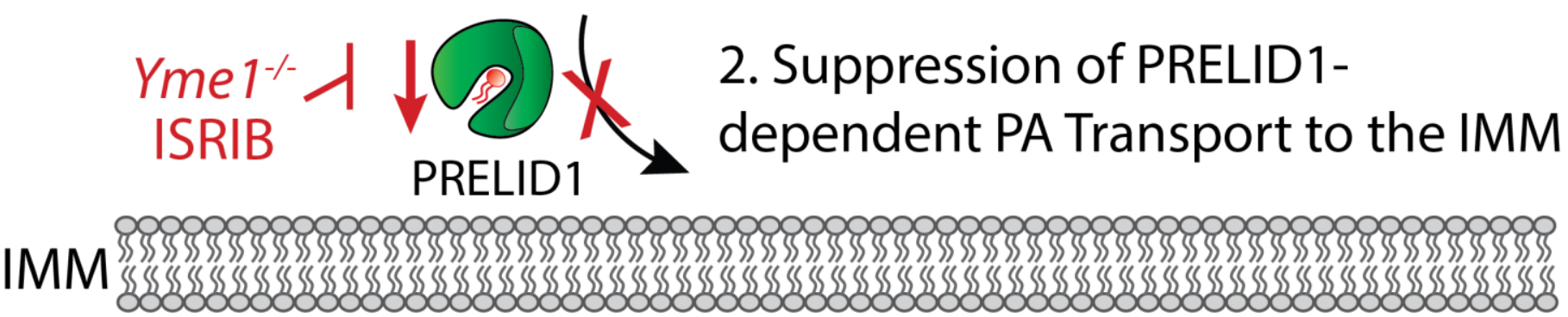

Figure 6. Proposed mechanism for PERK-regulated mitochondrial elongation during ER stress. In response to ER stress, PERK is activated, resulting in an increase in total and mitochondrial PA that was previously suggested to result from PERK kinase-dependent phosphorylation of diacylglycerol (DAG) to produce PA. ${ }^{52}$ YME1L degrades the intramitochondrial PA transporter PRELID1 downstream of PERKdependent translational attenuation, limiting the trafficking of PA to the inner mitochondrial membrane (IMM) and resulting in the accumulation of PA on the outer mitochondrial membrane (OMM). This increase in OMM PA then promotes mitochondrial elongation by inhibiting mitochondrial fission, likely through the previously reported direct inhibition of DRP1 activity. ${ }^{51}$ Specific manipulations employed to manipulate specific steps in this pathway are shown. 
bioRxiv preprint dol: https://doi.org/10.1101/2022.02.23.481593; this version posted February 23,2022 . The copyright holder for this preprint

(which was not certified by peer review) is the author/funder, who has granted bioRxiv a license to display the preprint in perpetuity. It is made

available under aCC-BY 4.0 International license.

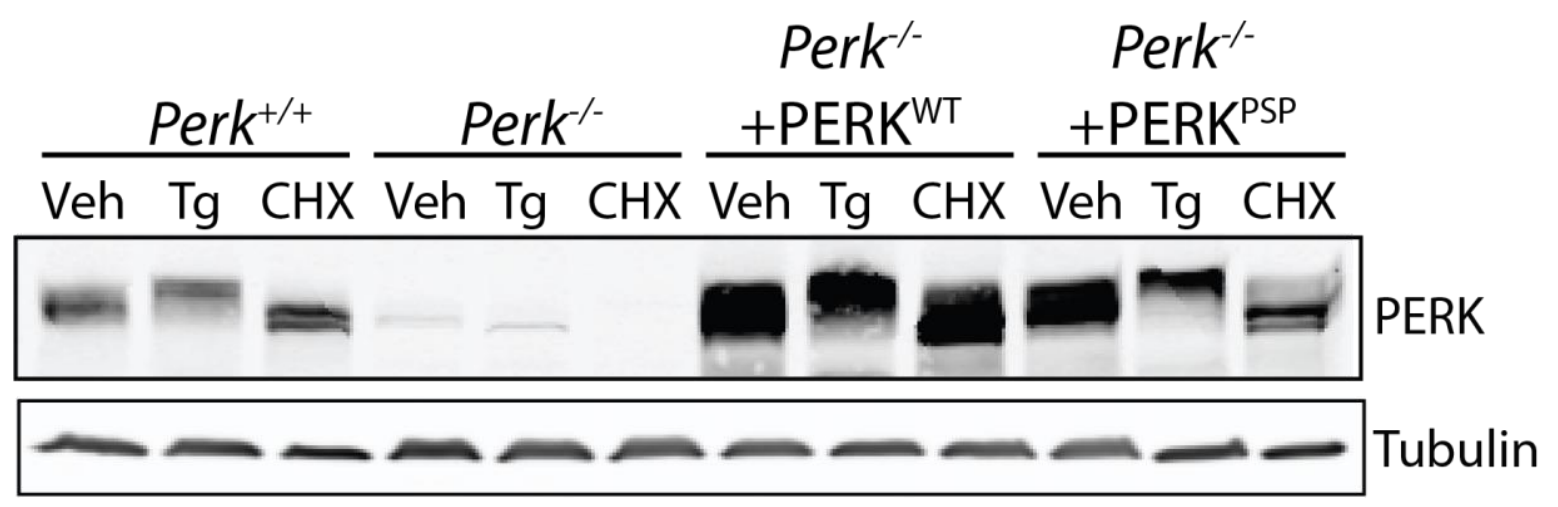

Figure S1 (Supplement to Figure 1. ER stress induced mitochondrial elongation is impaired in cells expressing hypomorphic PERK variants). Immunoblot of lysates prepared from Perk ${ }^{+/+}$MEFs, Perk ${ }^{-/}$MEFs, or Perk ${ }^{-1-}$ MEGs transected with wild-type PERK ${ }^{\mathrm{WT}}$ or the PSP-associated PERK allele (PERK ${ }^{\text {PST }}$ ) treated for 6 $\mathrm{h}$ with thapsigargin $(\mathrm{Tg} ; 500 \mathrm{nM})$ or cycloheximide $(\mathrm{CHX} ; 50 \mu \mathrm{g} / \mathrm{mL})$. 

available under aCC-BY 4.0 International license.
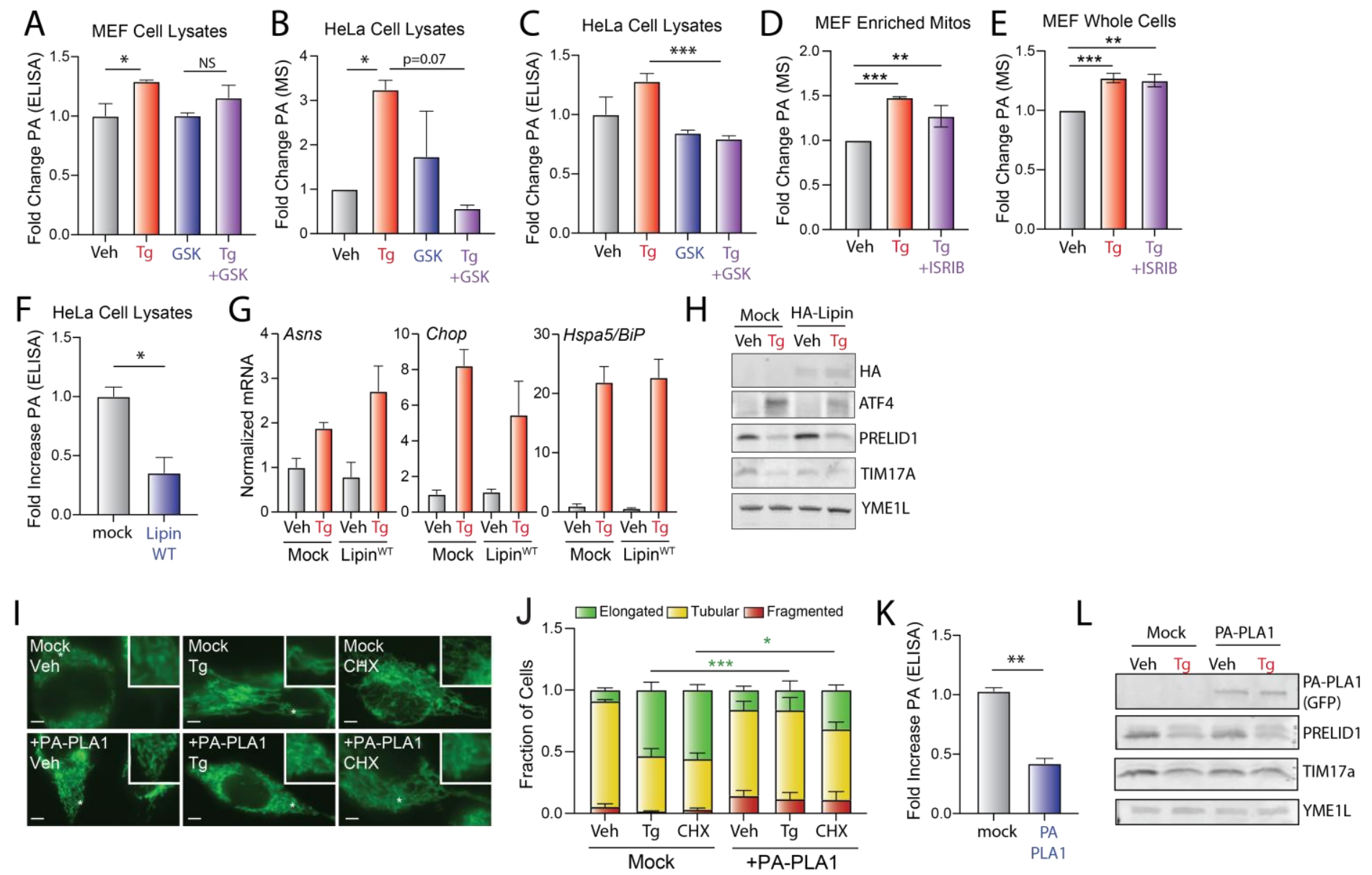

Figure S2 (Supplement to Figure 2. Overexpression of PA lipases inhibits ER stress induced mitochondrial elongation.). A. Relative phosphatidic acid (PA), measured by ELISA (see Materials and Methods), in whole cell extracts isolated from MEF cells treated for $3 \mathrm{~h}$ with vehicle, thapsigargin ( $\mathrm{Tg}, 500 \mathrm{nM})$, and/or GSK2606414 $(1 \mu \mathrm{M})$. Error bars show SEM for $n=3$ replicates ${ }^{*} p<0.05$ for unpaired t-test. B,C. Relative PA, measured by mass spectrometry $(\mathbf{B})$ or ELISA $(\mathbf{C})$, in lysates prepared from HeLa cells treated for $3 \mathrm{~h}$ with vehicle, thapsigargin $(\mathrm{Tg}, 500 \mathrm{nM})$, and/or GSK2606414 $(1 \mu \mathrm{M})$. Error bars show SEM for $\mathrm{n}=3$ replicates ${ }^{*} p<0.05$ for paired t-test in (B) and ${ }^{* * *} p<0.005$ for unpaired t-test in (C). D,E. Relative PA levels, measured by mass spectrometry, in mitochondrial enriched fractions or whole lysates from MEF cells treated for $3 \mathrm{~h}$ with vehicle, thapsigargin $(\mathrm{Tg} ; 500 \mathrm{nM})$, and ISRIB $(0.2 \mu \mathrm{M})$. Error bars show SEM for $\mathrm{n}=3$ replicates. ${ }^{* *} \mathrm{p}<0.01$, ${ }^{* * *} p<0.005$ for paired t-test. F. Relative PA, measured by ELISA, in lysates of HeLa cells expressing mock or Lipin $^{\text {WT }}$. Error bars show SEM for $n=3$ replicates. ${ }^{*} p<0.05$ for unpaired. G. Expression, measured by $q P C R$, of Asns, Chop, and Hspa5/BiP in HeLa cells expressing mock or Lipin ${ }^{\mathrm{WT}}$ treated for $3 \mathrm{~h}$ with vehicle or thapsigargin $(\mathrm{Tg}, 500 \mathrm{nM})$. H. Immunoblot of lysates prepared form HeLa cells expressing mock or Lipin WT treated for $3 \mathrm{~h}$ with vehicle or thapsigargin (Tg; $500 \mathrm{nM}$ ). Note that the Lipin construct is HA tagged allowing detection with the HA antibody. I. Representative images of HeLa cells expressing ${ }^{\mathrm{mt}} \mathrm{GFP}$ transfected with mock or GFP-tagged PA-PLA1 then treated for $3 \mathrm{~h}$ with thapsigargin (Tg; $500 \mathrm{nM})$ or cycloheximide (CHX; 50 $\mu \mathrm{g} / \mathrm{mL}$ ). The inset shows 2-fold magnification of the image centered on the asterisk. Scale bars, $5 \mu \mathrm{m}$. Note that the presence of GFP on PA-PLA1 did not influence our ability to monitor mitochondrial morphology in these cells. J. Quantification of fragmented (red), tubular (yellow), or elongated (green) mitochondria from the images shown in (I). Error bars show SEM for $n=3$ experiments. ${ }^{*} p<0.05$, ${ }^{* * *} p<0.005$ for 2 -way ANOVA (green indicates comparisons between elongated mitochondria fractions). K. Relative PA, measured by ELISA, in lysates of HeLa cells expressing mock or PA-PLA1. Error bars show SEM for $n=3$ replicates. Error bars show SEM for $\mathrm{n}=3$ replicates. ${ }^{* *} \mathrm{p}<0.01$ for unpaired t-test. L. Immunoblot of lysates prepared form HeLa cells expressing mock or PA-PLA1 treated for $3 \mathrm{~h}$ with vehicle or thapsigargin $(\mathrm{Tg} ; 500 \mathrm{nM})$. 

available under aCC-BY 4.0 International license.
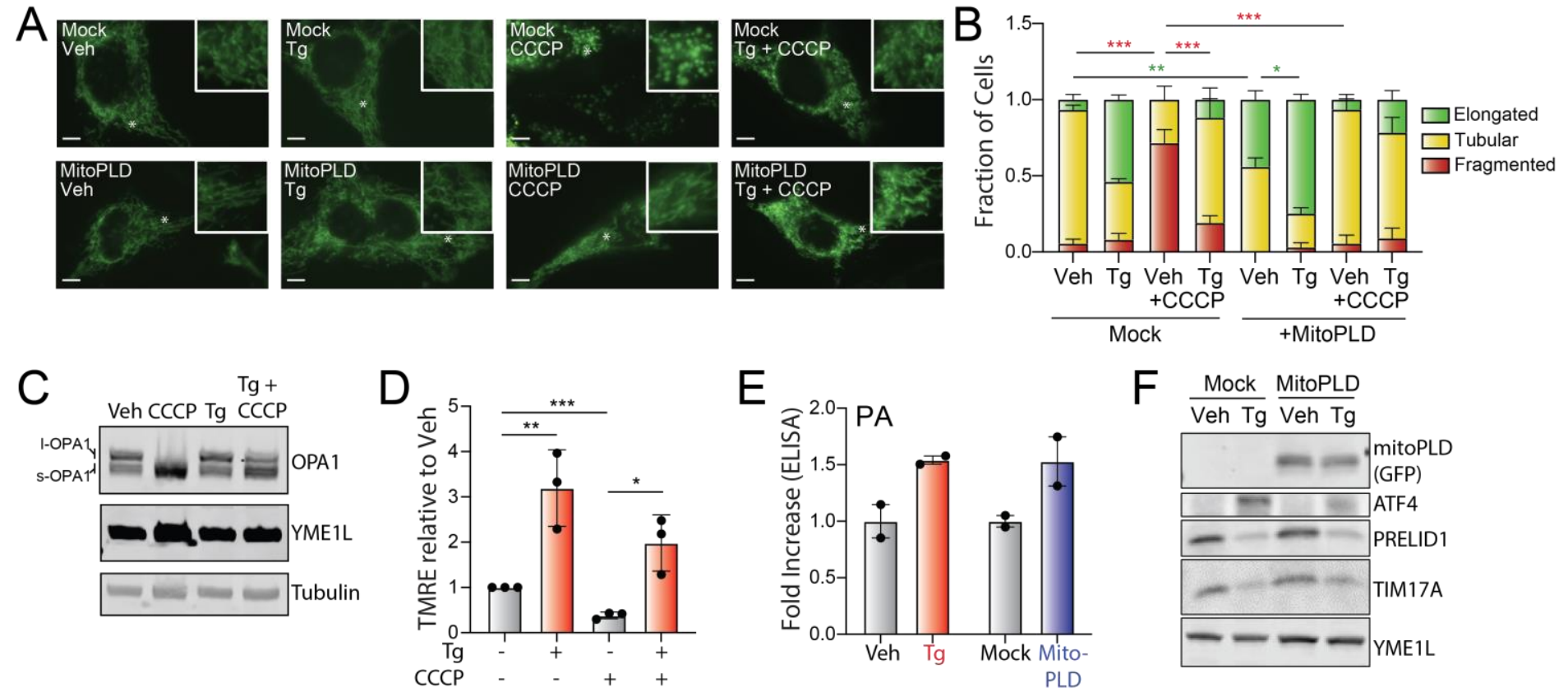

Figure S3 (Supplement to Figure 3. ER stress induced mitochondrial elongation inhibits lonomycininduced mitochondrial fragmentation.) A. Representative images of HeLa cells expressing ${ }^{\mathrm{mt}} \mathrm{GFP}$ transfected with mock or mitoPLD ${ }^{\text {GFP }}$ and then pretreated for $3 \mathrm{~h}$ with thapsigargin $(\mathrm{Tg} ; 500 \mathrm{nM})$ followed by a 30 min incubation with CCCP $(1 \mu \mathrm{M})$, as indicated. The inset shows 2 -fold magnification of the image centered on the asterisk. Scale bars, $5 \mu \mathrm{m}$. Note that the expression of the mitoPLD GFP did not impair our ability to monitor mitochondrial morphology in these cells. B. Quantification of fragmented (red), tubular (yellow), or elongated (green) mitochondria from the images shown in (A). Error bars show SEM for $n=3$ experiments. $p$ value reflects comparisons of elongated (green) or fragmented (red) mitochondria populations for the indicated conditions. ${ }^{*} p<0.05,{ }^{* *} p<0.01,{ }^{* *} p<0.005$ for 2 -way ANOVA (red indicates comparison between fragmented mitochondria fractions; green indicates comparisons between elongated mitochondria fractions). C. Immunoblot of lysates prepared from MEF ${ }^{\text {mtGFP }}$ cells pre-treated for $3 \mathrm{~h}$ with thapsigargin $(\mathrm{Tg} ; 500 \mathrm{nM})$ then challenged with CCCP for $30 \mathrm{~min}$. D. Mitochondrial polarization, measured by TMRE fluorescence, in MEF cells pre-treated for $3 \mathrm{~h}$ with thapisgargin $(\mathrm{Tg} ; 500 \mathrm{nM})$ then challenged for 30 min with CCCP $(10 \mu \mathrm{M})$. Error bars show SEM for $n=3$ replicates. ${ }^{*} p<0.05$, ${ }^{* *} p<0.01,{ }^{* *} p<0.005$ for paired t-tests. E. Phosphatidic acid (PA), measured by ELISA, in HeLa cells treated with thapsigargin (Tg; $500 \mathrm{nM}, 3 \mathrm{~h}$ ) or expressing mock or mitoPLD. Error bars show SEM for $\mathrm{n}=2$ replicates. Individual replicates are shown. F. Immunoblot of lysates prepared om HeLa cells transfected with mock or mitoPLD and treated for $3 \mathrm{~h}$ with vehicle or thapsigargin (Tg; $500 \mathrm{nM})$. Note mitoPLD is tagged with GFP allowing detection of this protein with the GFP antibody. 

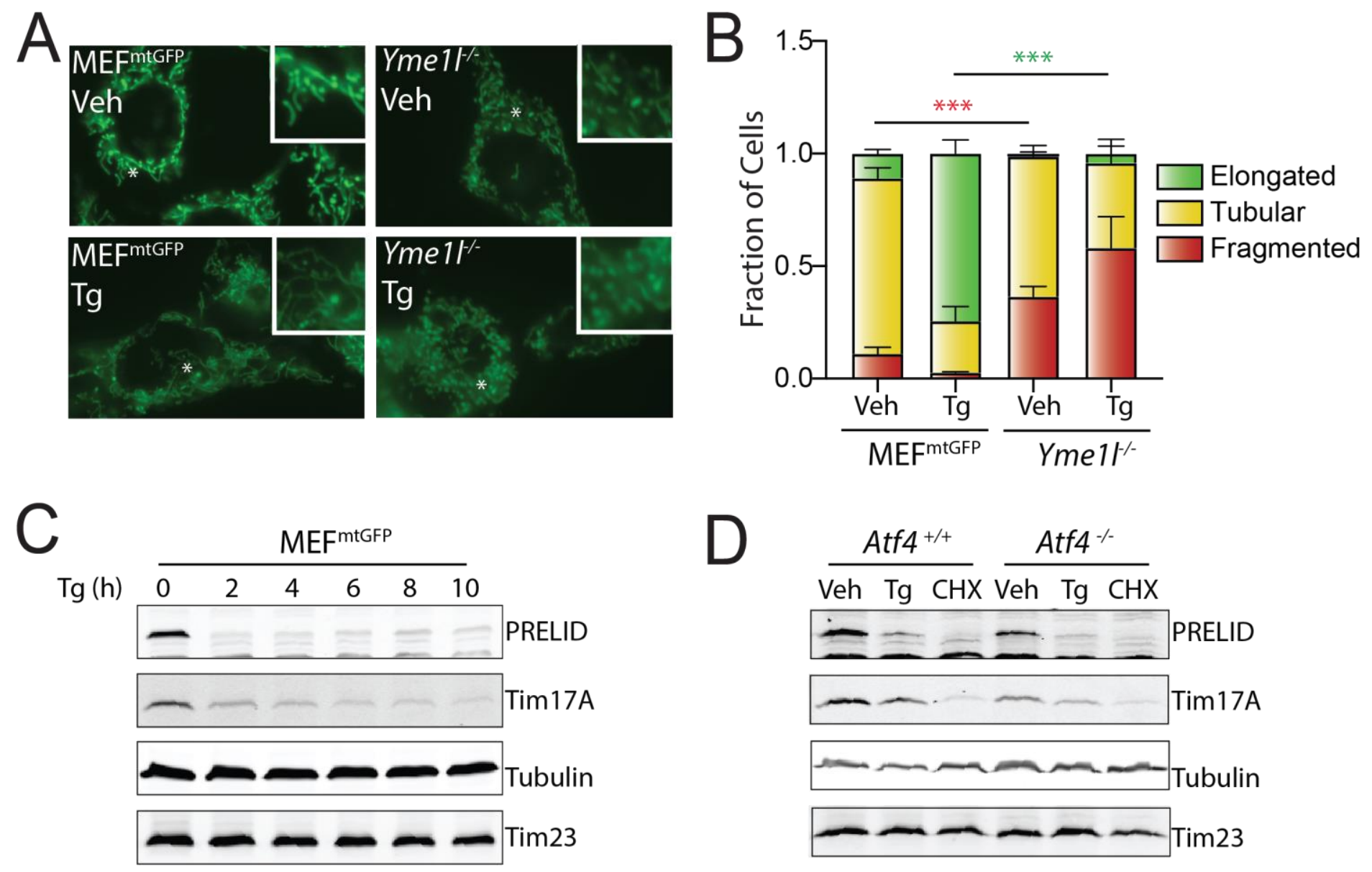

D

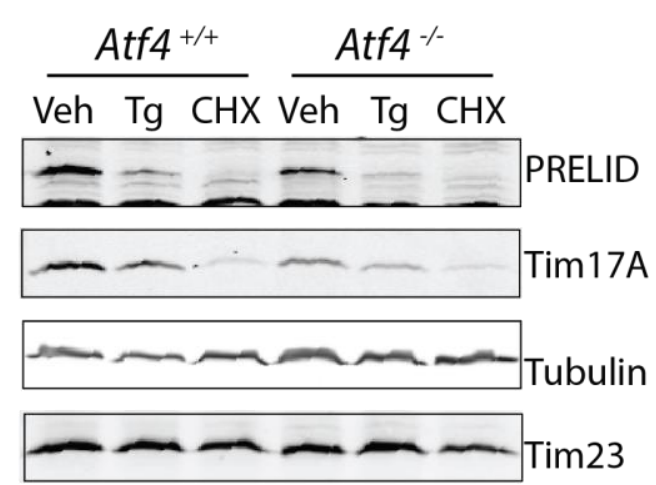

Figure S4 (Supplement to Figure 4. ER stress reduces PRELID1 through a YME1L-dependent mechanism downstream of PERK-dependent translational attenuation). A. Representative images of MEF $^{\text {mtGFP }}$ cells and Yme1/-deficient MEF ${ }^{\text {mtGFP }}$ cells treated for $6 \mathrm{~h}$ with thapsigargin (Tg; $\left.50 \mathrm{nM}\right)$. The inset shows 2-fold magnification of the image centered on the asterisk. Scale bars, $5 \mu \mathrm{m}$. B. Quantification of fragmented (red), tubular (yellow), or elongated (green) mitochondria from the images shown in (A). Error bars show SEM for $n=7$ experiments. $p$-value reflects comparisons of elongated (green) or fragmented (red) mitochondria populations for the indicated conditions. ${ }^{* * *} \mathrm{p}<0.005$ for 2 -way ANOVA (red indicates comparison between fragmented mitochondria fractions; green indicates comparisons between elongated mitochondria fractions). C. Immunoblot of lysates prepared from MEF ${ }^{\text {mtGFP }}$ cells treated with thapsigargin (Tg; $\left.500 \mathrm{nM}\right)$ for the indicated time. D. Immunoblot of lysates prepared from $\mathrm{Atf4}^{+/+}$and $\mathrm{Atf4}^{-/-} \mathrm{MEFs}$ treated with thapsigargin (Tg; $500 \mathrm{nM})$ or $\mathrm{CHX}(50 \mu \mathrm{g} / \mathrm{mL})$ for $3 \mathrm{~h}$. 
bioRxiv preprint doi: https://doi org/10.1101/2022 $02.23 .481593 \cdot$ this version posted February 23,2022 . The copyright holder for this preprint (which was not certified by peer review) is the author/funder, who has granted bioRxiv a license to display the preprint in perpetuity. It is made available under aCC-BY 4.0 International license.

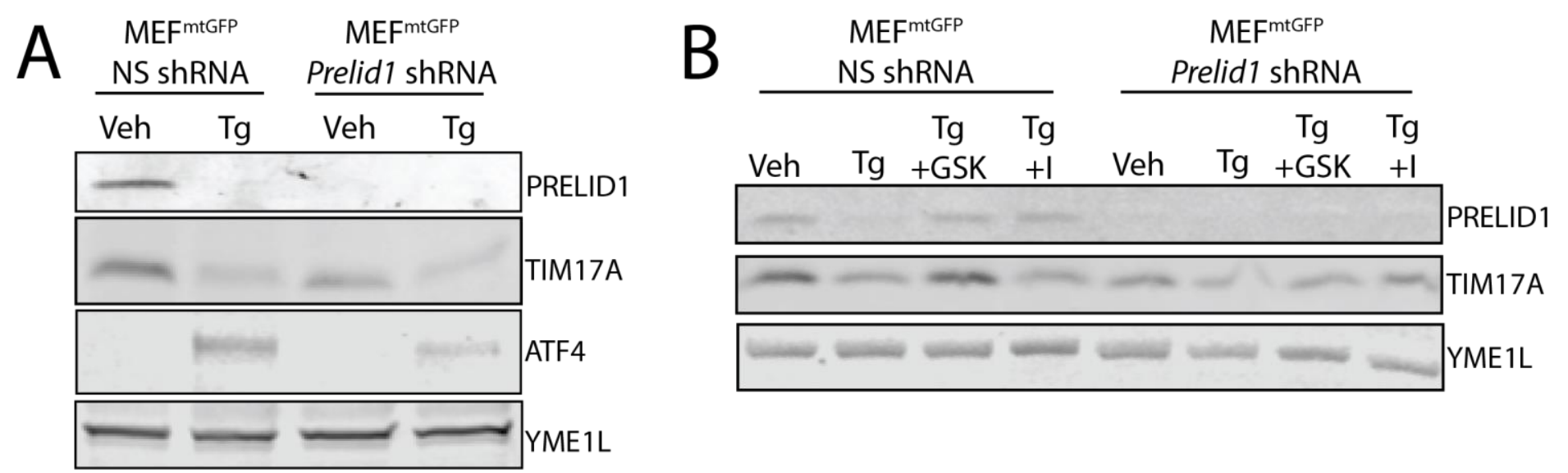

Figure S5 (Supplement to Figure 5. Reductions in PRELID1 contribute to ER stress induced mitochondrial elongation.) A. Immunoblot of lysates prepared from MEF ${ }^{\text {mtGFP }}$ expressing non-silencing (NS) or Prelid1 shRNA and treated for $3 \mathrm{~h}$ with vehicle or thapsigargin (Tg, $500 \mathrm{nM})$. B. Immunoblot of lysates prepared from HeLa cells expressing non-silencing (NS) or PRELID1 shRNA and treated for $3 \mathrm{~h}$ with vehicle or thapsigargin ( $\mathrm{Tg}, 500 \mathrm{nM})$. 